\title{
Conservation status of North Pacific right whales
}

\author{
Robert L. Brownell, JR. *, Phillip J. Clapham ${ }^{+}$, Tomio Miyashita ${ }^{++}$and Toshio Kasuya ${ }^{\#}$
}

\begin{abstract}
The North Pacific right whale (Eubalaena japonica) is among the most endangered of all great whales, having been subject to intensive commercial whaling in the $19^{\text {th }}$ century. All available $20^{\text {th }}$ century records of this species in the North Pacific were reviewed. There has been a total of 1,965 recorded sightings since 1900; of these, 988 came from the western North Pacific, 693 from the eastern North Pacific and 284 had no location specified. Thirteen strandings (all but one from the western North Pacific) were recorded. Known catches for commercial or scientific purposes totalled 742 (331 in the western North Pacific, 411 in the eastern North Pacific). Most of the reported Soviet 'sightings' in the eastern North Pacific were actually catches, as may be the case for Soviet sightings in the Okhotsk Sea. In addition, the impact of known Soviet illegal catches in the Okhotsk Sea may be reflected in an apparent decline in sightings after the 1960s (although this may be partly explained by low observer effort). Overall, the data support the hypothesis that at least two stocks of right whales exist in the North Pacific. Any recovery in the western North Pacific population was compromised by the Soviet catches in the Okhotsk region, although recent sightings suggest that this population is still large enough to sustain reproduction. By contrast, Soviet catches in the now-smaller eastern North Pacific population have severely reduced its prospects for recovery. Although the prognosis for this population is poor, a long-term monitoring programme is required to better understand its conservation status and to determine whether it may be affected by human-related problems that would require mitigation.
\end{abstract}

KEYWORDS: RIGHT WHALE; PACIFIC OCEAN; NORTHERN HEMISPHERE; WHALING-MODERN; WHALING-HISTORICAL; CONSERVATION; DISTRIBUTION

\section{INTRODUCTION}

The North Pacific right whale, Eubalaena japonica, was once abundant in much of the North Pacific. The crew of the American whaler Ganges, one of the first vessels to work in the so-called 'Northwest Ground' (the Gulf of Alaska region), reported seeing 'millions' of right whales in 1835-36 (Webb, 1988). Contemporary records, compiled from the logs of $19^{\text {th }}$ century whaling ships by the American naval officer Matthew Fontaine Maury, reveal an abundance of right whales across a broad range that stretched from Alaska to Japan (Scarff, 1986a; 1991). However, intensive commercial whaling proved devastating, and today the North Pacific right whale populations are among the most endangered of all the great whales (IWC, 2001b).

Aboriginal whaling for this species was conducted by various peoples from Washington state and British Columbia to eastern Asia (Tomilin, 1957; Mitchell, 1979; Omura, 1986; Scarff, 1991; Mitchell and Reeves, 2001). However, it does not appear that right whales were usually the primary target of these hunts, nor were they taken in significant numbers. Right whales were taken with some regularity in coastal Japan, where net whaling began in the $17^{\text {th }}$ century (Omura, 1986). Net whaling also occurred for a short time off southern Sakhalin Island prior to 1904 (Tomilin, 1957). Catch records from these fisheries are incomplete so it is not known whether this method of exploitation had any significant effects on the population.

By contrast, the impact of commercial whaling was rapid and devastating. Pelagic whaling for right whales started first in the Sea of Japan during the 1820s. From 1835, from what came to be known as the Kodiak or Northwest Ground, whaling effort quickly spread across the Pacific. By 1845 , American vessels were operating in the southern Okhotsk Sea (Webb, 1988; Scarff, 1991). By 1847-48, catches of right whales had already declined, and the discovery of bowhead whales (Balaena mysticetus) in high latitudes north of the Bering Strait resulted in a change of focus for the majority of vessels in the American fishery. Best (1987) estimated that American pelagic whalers in the North Pacific (including the Okhotsk Sea but not the Sea of Japan) killed an estimated 14,500 right whales. These catches do not make any allowance for hunting loss and do not include catches by British, French and other European whalers. The total North Pacific catch of right whales has been variously estimated at 20,000 (Du Pasquier, 1986), or between 26,500 and 37,000 (Scarff, 2001). Right whales continued to be taken after 1849 , although the paucity of catch records after 1900 is testament to the damage inflicted upon these populations during the period of extensive exploitation. The species was never again the principal focus of commercial whaling. Although a Japanese coastal fishery, using modern methods, took 192 right whales in the western North Pacific between 1900 and 1948 (Omura, 1986), documented legal catches elsewhere for either commercial or scientific purposes were infrequent.

The Convention for the Regulation of Whaling was concluded in Geneva on 24 September 1931. Article 4 of the Convention states that 'The taking or killing of right whales, which shall be deemed to include North-Cape whales, Greenland whales, southern right whales, Pacific right whales, and southern pigmy right whales, is prohibited'. After the close of the meeting numerous whaling nations ratified the Convention but Japan and the USSR were not parties to the Convention. After World War II, Japan was allowed to conduct whaling operations again under the regulations established by the Supreme Commander for the Allied Powers in 1945. One of these regulations stated that the killing of gray and right whales in the North Pacific was prohibited. Japan joined the IWC in April 1951. The USSR joined the IWC in 1948. Furthermore, it is now known that the Soviet Union conducted illegal whaling on a large scale, beginning in the Southern Hemisphere in the 1949/50 season (Zemsky et al., 1995) and that this included large numbers of

\footnotetext{
* Southwest Fisheries Science Center, P.O. Box 271, La Jolla, CA 92038, USA.

${ }^{+}$Northeast Fisheries Science Center, 166 Water Street, Woods Hole, MA 02543, USA

++ National Far Sea Fisheries Research Institute, 5-7-1 Orido, Shimizu 424, Japan.

\# Faculty of Bioresources, Mie University, Kamihama-cho, Tsu, Mie 514-8507, Japan.
} 
right whales (Tormosov et al., 1998); related operations subsequently took hundreds of right whales in the Okhotsk Sea, around the Kuril Islands, in the southeastern Bering Sea and the Gulf of Alaska and maybe beyond (Yablokov, 1994; Doroshenko, 2000). The species has been so rare in the eastern North Pacific that, over the past forty years, even single sightings have merited publication (Gilmore, 1956; Woodhouse and Strickley, 1982; Carretta et al., 1994; Rowlett et al., 1994; Gendron et al., 1999). In the western portion of their former range, right whales have been observed with greater frequency in the Okhotsk Sea (Miyashita and Kato, 1998) but sightings elsewhere are still comparatively rare.

Much is unknown or uncertain regarding even some basic aspects of the biology and behaviour of the North Pacific right whale. There is no agreement on the number of populations that exist, the current population size is unknown for either the eastern or western population, and the location of the calving grounds remains a mystery. Indeed, with the exception of a series of Soviet and Japanese papers, based primarily on 23 animals killed for research purposes (Omura, 1958; Klumov, 1962; Omura et al., 1969), virtually nothing has been published on the biology of the species.

The purpose of this paper is to review knowledge concerning the right whale in the North Pacific, and to attempt to assess the status of those animals that remain. For the purpose of conservation, it is important to take a basin-wide approach to understanding population structure and abundance. A complete listing of all known sightings, strandings and catches in the $20^{\text {th }}$ century is provided.

\section{METHODS AND MATERIALS}

All available records of North Pacific right whales from the $20^{\text {th }}$ century were reviewed. Scarff $(1986 a ; 1991)$ provided a thorough analysis of the right whale's historic distribution as determined from $19^{\text {th }}$ century whaling manuscripts collected by Maury $(1852 ; 1853)$ and additionally summarised sighting records for the eastern North Pacific since 1855. The current paper is based on a complete listing of all known sightings, strandings or catches of right whales, including numerous published and unpublished sources that were either not covered by, or were unavailable to, Scarff. The review begins in 1900 because it represents a convenient division and the beginning of modern-type whaling in the North Pacific (Tonnessen and Johnsen, 1982).

There is sometimes confusion in records between the right whale and the closely related bowhead. For example, we believe that the right whales referred to by Tomilin (1957) were bowheads, since their reported distribution and timing of occurrence in the western Okhotsk Sea closely fits known patterns for this species. Similarly, it is clear that all of the right whales reported by Klumov (1962) in the vicinity of the Shantar Islands were in fact bowheads (Brownell, unpublished). The Russian language contains words specific to each species (ЯПонСкИй кíт =E. glacialis/E. japonica; and ГренландскИй кі́т = B. mysticetus), as well as a more generic term ('smooth whales') which can imply either. In cases where the species identity was in question, the original texts were examined to verify the reliability of the English translation. The Russian originals were also examined when two or more papers gave conflicting accounts of the same sighting event(s). Where possible, authors (both Japanese and Russian) were queried for clarification of confusing or contradictory data.
In certain cases, numbers have been extrapolated from plots in the original papers, notably Berzin and Rovnin (1966) and Omura et al. (1969). It should be noted that it is not possible to obtain a precise count from these sources. In the case of Omura et al. (1969), one of us (TK) obtained the original data (those for the period 1959-62) on which some of this paper was based. Since these data give more detail in terms of sighting dates, locations and numbers of whales they have been incorporated into this review. For the remainder of the plotted sightings in Omura et al. (1969), each plot has been counted as a single whale, although it is known from the detailed data that this is not always the case ${ }^{1}$. Similarly, the two types of symbol used in the plots of Berzin and Rovnin (1966, fig. 6) represent sightings of either ' $1-3$ ' or ' $15-20$ ' animals; consequently, calculations from counts of these plots yield a range for each area rather than a single total. In all cases where a sighting has been reported as a range, the midpoint of that range has been used when calculating the total number of sightings for each area.

\section{Sources and effort}

The majority of records of North Pacific right whales come from vessels associated with the whaling industry. These include incidental sightings by catcherboats, factory ships and marking cruises, as well as much more extensive records provided by scouting boats or by research vessels engaged in directed sighting surveys for the specific purpose of establishing the abundance and distribution of whales. Of particular value is the series of extensive annual surveys conducted since 1964 by Japan. These surveys have covered virtually the entire North Pacific north of $20^{\circ} \mathrm{N}$, although largely confined to the summer months. Their effort and results are summarised by Wada (1975) and by the annual Japanese national progress reports on whale research to the International Whaling Commission from 1976 onwards.

Almost all other sources of sightings are incidental in nature. They range from scientific research vessels (notably those of the Soviet Union) and recent aerial surveys off the west coast of the USA, to opportunistic sightings from a variety of vessels and individuals.

With the exception of the Japanese sighting surveys, it is generally not possible to meaningfully quantify the effort invested by these various sources. However, some general remarks concerning coverage are possible, and these are incorporated into the Discussion.

\section{Study area and regional divisions}

The study area includes the entire North Pacific Ocean from the equator northwards, including bodies of water outside the Pacific proper (e.g. Bering Sea, Okhotsk Sea). Since there is disagreement regarding the number and boundaries of right whale stocks in the North Pacific, the study area was arbitrarily divided into eastern and western halves at the $180^{\circ}$ line of longitude.

In addition to listing all records chronologically within event type (sighting, catch, stranding), a record was also assigned to a general region. Regional divisions are necessarily somewhat arbitrary, and are used solely for the purpose of more clearly summarising the data. The principal divisions of the North Pacific used here can be broadly defined as follows.

\footnotetext{
1 There is an error in table 14 of Omura et al. (1969). The 'pelagic' sighting totals reported for the years 1954-57, which are based upon table 2 from Omura (1958), are incorrect. They represent the total of pelagic plus coastal sightings from Omura (1958). Pelagic data in table 14 should read: 1954 (24), 1955 (8), 1956 (75) and 1957 (25).
} 
Japan

The waters within approximately 200 miles of the Japanese coast, including outlying islands such as the Amamis (Amami Ōshima) and the Bonins (Ogasawara Guntō). However, the region around the southwestern Kuril Islands is excluded.

\section{Kurils}

Any area in the immediate vicinity of the Kuril Islands, whether on the Okhotsk Sea or Pacific side.

\section{Okhotsk Sea}

Any region within the Okhotsk Sea, including Sakhalin Island, but excluding the Kurils.

\section{Aleutians}

Any area within a hundred nautical miles of the Aleutian Islands, either the Bering Sea or Pacific side. The Aleutians are divided into eastern and western halves, separated by the $180^{\circ}$ line of longitude.

\section{NW Pacific}

Any offshore waters (further than approximately 200 miles from land) west of $180^{\circ}$, including the Commander Islands.

\section{NE Pacific}

Any offshore waters (further than 100 miles from land) east of $180^{\circ}$, within the exception of the Northwest Ground as defined below.

\section{West Coast}

Waters off the western coast of North America, from British Columbia to Baja California, within 100 miles of shore.

\section{Northwest Ground}

This name is borrowed from the whaling literature, since it nicely describes an important area for which there is otherwise no convenient name. It has been arbitrarily defined as extending from the Gulf of Alaska south to $50^{\circ} \mathrm{N}$. Waters to the south of this area are considered NE Pacific.

\section{Bering Sea}

Any portion of the Bering Sea except for those waters within 100 miles of the Aleutian Islands or the Alaska Peninsula. (Nineteenth century whalemen developed their own terminology for the North Pacific whaling grounds. They divided the Bering Sea into four major sections: Bristol Bay, Kamchatka Sea, Anadyr Sea and the Bering Straits. However, these are not used here).

\section{Hawaii}

Any area within the vicinity of the Hawaiian Island chain.

\section{RESULTS}

The total number of sightings, commercial takes, strandings and incidental catches since 1900 are summarised for both the eastern and western North Pacific in Table 1. It should be noted that, while we have been careful to exclude cases where two reports have either clearly or probably used the same data, no allowance for possible resightings of individual animals at different times can be made in the sighting records. Totals reported here are necessarily approximate. On the one hand, cases of duplication will inflate the number of individual whales observed; conversely, some sightings counted here as single animals probably represent two or more whales, thus negatively biasing our totals.

Table 1

Total numbers of sightings, catches and strandings/entanglements of right whales recorded in the North Pacific between 1900 and 1999. For the purpose of this paper, the boundary between the western and eastern regions is set at the $180^{\circ}$ line of longitude.

\begin{tabular}{lcccr}
\hline Region & Sightings & Catches & $\begin{array}{c}\text { Strandings or } \\
\text { entanglements }\end{array}$ & Total \\
\hline Western North Pacific & 988 & 331 & 12 & 1,331 \\
Eastern North Pacific & 693 & 411 & 1 & 1,105 \\
Area not specified & 284 & - & - & 284 \\
Total & 1,965 & 742 & 13 & 2,720 \\
\hline
\end{tabular}

\section{Sightings}

Excluding animals that were either stranded or taken by whalers, there have been 1,965 sightings $^{2}$ of living right whales in the North Pacific since 1900. Of these, 988 were reported from the western portions of this ocean basin and 693 from the eastern (Tables 2.1 and 2.2). There were an additional 284 sightings of right whales by Japanese and Soviet vessels with the location described only as 'North Pacific', without reference to area (Table 2.3). Although all but twenty of these are plotted in several figures by Omura et al. (1969), it is largely impossible to count the number of sightings in each half of the region since the plots represent an amalgamation of data from several sources and time periods; the exceptions are sightings from the period 1959-62, for which original data were available, as noted above. In addition, a few sightings have no or confusing numbers associated with them (e.g. the sightings from 1948 reported by Sleptsov, 1952), and these are not included in the totals.

Sightings are summarised by area in Table 2.4. In the western North Pacific, three areas accounted for more than $90 \%$ of the 988 sightings: Japanese waters (370 sightings, or $37.4 \%$ ); the Kuril Islands (331 sightings, 33.5\%); and the Okhotsk Sea (195 sightings, 19.7\%). Of the 693 sightings in the eastern North Pacific, the greatest number (269, or $38.8 \%$ ) were from the area of eastern Aleutians. More than a quarter $(177$, or $25.5 \%)$ were from the Northwest Ground; however, 139 of these sightings were derived from ranges given in the plots of Berzin and Rovnin (1966), and should therefore be interpreted with caution.

If one calculates the number of sightings on the Northwest Ground from the plots of Berzin and Rovnin (1966), one obtains a range of from 88 to 189 right whales observed by Soviet research vessels in the period 1958-1964 (the midpoint of this range, which we use for the totals given in Tables 1 and 2.4, is 139). These plots show right whales observed over a wide area within this region; the text of this paper notes that 200 right whales were encountered in 1963 in all of the northeastern Pacific. By contrast, Berzin and Doroshenko (1982) give a much more specific location on the Northwest Ground $\left(51^{\circ} \mathrm{N} 145^{\circ} \mathrm{W}\right)$ for a single sighting of 200 right whales in 1963000 , made by the same Soviet research vessels.

\section{Text continues on p. 278}

\footnotetext{
2 This total ignores certain and probable duplicates, and counts the sighting of '40-45' whales by Kuzmin and Berzin (1975) as 43. It uses the midpoints of the various ranges calculated from fig. 6 of Berzin and Rovnin (1966); these total 20 and 324 for the western and eastern North Pacific, respectively.
} 
Table 2.1

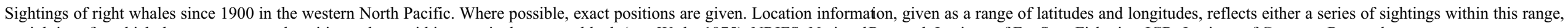
or sightings for which the source reported position only to within a particular survey block (e.g. Wada, 1975). NRIFS=National Research Institute of Far Seas Fisheries; ICR=Institute of Cetacean Research.

\begin{tabular}{|c|c|c|c|c|c|c|}
\hline Date & Latitude & Longitude & Location & No. & Source & Remarks \\
\hline May 1931 & & & Off Akkeshi, eastern Hokkaido & 9 & Shino, 1932 & 12 whales sighted, but 3 were killed (see Table 3.1 ) \\
\hline Apr. 1941-1957 & & & East and southeast of Hokkaido, Japan & 37 & Omura, 1958 & Japanese catcherboat sightings \\
\hline \multirow[t]{2}{*}{ May 1941-1957 } & & & East and southeast of Hokkaido, Japan & 46 & Omura, 1958 & Japanese catcherboat sightings \\
\hline & & & Lat $46 / 47$ east of Kurils & 3 & & \\
\hline \multirow[t]{3}{*}{ Jun. 1941-1957 } & & & SE of Hokkaido, Japan & 4 & Omura, 1958 & Japanese catcherboat sightings \\
\hline & & & SE of Paramushir I., Kurils & 2 & & \\
\hline & & & S of Commander Islands & 10 & & \\
\hline \multirow[t]{3}{*}{ Jul. 1941-1957 } & & & SE of Hokkaido, Japan & 2 & Omura, 1958 & Japanese catcherboat sightings \\
\hline & & & SE of Paramushir I., Kurils & 3 & & \\
\hline & & & SE of Kamchatka & 2 & & \\
\hline Aug. 1941-1957 & $52^{\circ} \mathrm{N}$ & $163^{\circ} \mathrm{E}$ & SE of Kamchatka & 2 & Omura, 1958 & Japanese catcherboat sightings \\
\hline 1948 & & & Northeast Okhotsk Sea & ? & Sleptsov, 1952 & Cited in Tomilin (1957) as 'small groups of from 2-3 to 15 animals' \\
\hline Apr.-Nov. 1951 & $39-52^{\circ} \mathrm{N}$ & $140-158^{\circ} \mathrm{E}$ & Kuril Is (both Okhotsk Sea and Pacific sides) & 57 & Klumov, 1962 & Russian catcherboat sightings \\
\hline Apr.-Nov. 1953 & $39-52^{\circ} \mathrm{N}$ & $140-158^{\circ} \mathrm{E}$ & Kuril Is (both Okhotsk Sea and Pacific sides) & 16 & Klumov, 1962 & Russian catcherboat sightings \\
\hline Apr.-Nov. 1954 & $39-52^{\circ} \mathrm{N}$ & $140-158^{\circ} \mathrm{E}$ & Kuril Is (both Okhotsk Sea and Pacific sides) & 6 & Klumov, 1962 & Russian catcherboat sightings \\
\hline Pre-1955 & & & NE Okhotsk Sea and western Kamchatka & ? & Sleptsov, 1955 & \\
\hline 1955 & & & Amami Is, Japan & 1 & Miyazaki and Nakayama, 1989 & Autumn \\
\hline Apr.-Nov. 1955 & $39-52^{\circ} \mathrm{N}$ & $140-158^{\circ} \mathrm{E}$ & Kuril Is (both Okhotsk Sea and Pacific sides) & 85 & Klumov, 1962 & Russian catcherboat sightings \\
\hline Apr.-Nov. 1956 & $39-52^{\circ} \mathrm{N}$ & $140-158^{\circ} \mathrm{E}$ & Kuril Is (both Okhotsk Sea and Pacific sides) & 50 & Klumov, 1962 & Russian catcherboat sightings \\
\hline Apr.-Nov. 1957 & $39-52^{\circ} \mathrm{N}$ & $140-158^{\circ} \mathrm{E}$ & Kuril Is (both Okhotsk Sea and Pacific sides) & 109 & Klumov, 1962 & Russian catcherboat sightings \\
\hline 1958-1964 & & & Western Aleutians & $10-30$ & Berzin and Rovnin, 1966 & Soviet sighting cruises. Shown (Fig. 6) as ten plots of ' $1-3$ ' whaks \\
\hline 1 Jun. 1959 & $53^{\circ} 42^{\prime} \mathrm{N}$ & $167^{\circ} 23^{\prime} \mathrm{E}$ & Western Aleutians & 1 & ICR data & Japanese catcherboat sighting \\
\hline 28 Jun. 1959 & $53^{\circ} 05^{\prime} \mathrm{N}$ & $179^{\circ} 33^{\prime} \mathrm{E}$ & Western Aleutians & 1 & ICR data & Japanese catcherboat sighting \\
\hline 1965 & & & 'Coastal' Japan & 102 & Omura et al., 1969 & Japanese catcherboat sightings \\
\hline \multicolumn{7}{|l|}{ and 1970} \\
\hline 1966 & & & ‘Coastal' Japan & 34 & Omura et al., 1969 & Japanese catcherboat sightings \\
\hline 1966 & $45-50^{\circ} \mathrm{N}$ & $160-170^{\circ} \mathrm{E}$ & NW Pacific, southeast of Kamchatka & 1 & Wada, 1975 & Japanese scoutboat sighting \\
\hline 1967 & & & ‘Coastal' Japan & 83 & Omura et al., 1969 & Japanese catcherboat sightings \\
\hline 1967 & & & Cape Terpeniya, eastern Sakhalin I. & 70 & Berzin and Vladimirov, 1989 & Seen by RV Vityaz \\
\hline 1967 & & & Okhotsk Sea & 14 & Berzin and Doroshenko, 1981 & Unclear; may be part of 70 reported by Berzin and Vladimirov (1989) \\
\hline 1967 & & & Okhotsk Sea side of Urup I. (Kurils) & ? & Berzin and Vladimirov, 1989 & 'Single individuals' \\
\hline Jul. 1968 & $48^{\circ} \mathrm{N}$ & $145-146^{\circ} \mathrm{E}$ & SE Sakhalin I., Okhotsk Sea & Few & Omura, 1968 & $\begin{array}{l}\text { Japanese catcher vessel sighting. Whales associated with those taken in } \\
\text { research catch; } 5 \text { or fewer whales. }\end{array}$ \\
\hline 1968 & $45-50^{\circ} \mathrm{N}$ & $160-170^{\circ} \mathrm{E}$ & NW Pacific, southeast of Kamchatka & 5 & Wada, 1975 & Japanese scoutboat sighting \\
\hline 1968 & $45-50^{\circ} \mathrm{N}$ & $170-180^{\circ} \mathrm{E}$ & NW Pacific, south of Aleutians & 1 & Wada, 1975 & Japanese scoutboat sighting \\
\hline 1968 & $50-55^{\circ} \mathrm{N}$ & $170-180^{\circ} \mathrm{E}$ & Aleutians, Pacific side & 1 & Wada, 1975 & Japanese scoutboat sighting \\
\hline 1969 & $40-45^{\circ} \mathrm{N}$ & $160-170^{\circ} \mathrm{E}$ & NW Pacific & 2 & Wada, 1975 & Japanese scoutboat sighting \\
\hline 1969 & $45-50^{\circ} \mathrm{N}$ & $160-170^{\circ} \mathrm{E}$ & NW Pacific, southeast of Kamchatka & 2 & Wada, 1975 & Japanese scoutboat sighting \\
\hline 1969 & $45-50^{\circ} \mathrm{N}$ & $170-180^{\circ} \mathrm{E}$ & South of Aleutians & 1 & Wada, 1975 & Japanese scoutboat sighting \\
\hline 1970 & $40-45^{\circ} \mathrm{N}$ & $150-160^{\circ} \mathrm{E}$ & NW Pacific, southeast of Kurils & 1 & Wada, 1975 & Japanese scoutboat sighting \\
\hline 1970 & $45-50^{\circ} \mathrm{N}$ & $160-170^{\circ} \mathrm{E}$ & NW Pacific, southeast of Kamchatka & 1 & Wada, 1975 & Japanese scoutboat sighting \\
\hline 1970 & $45-50^{\circ} \mathrm{N}$ & $170-180^{\circ} \mathrm{E}$ & South of Aleutians & 1 & Wada, 1975 & Japanese scoutboat sighting \\
\hline 1971 & & & Muroto, Shikoku, Kochi Prefecture, Japan & 1 & Kasamatsu and Miyashita, 1991 & Escaped from fishing gear \\
\hline 1971 & $45-50^{\circ} \mathrm{N}$ & $150-160^{\circ} \mathrm{E}$ & Near Kurils, Pacific side & 1 & Wada, 1975 & Japanese scoutboat sighting \\
\hline
\end{tabular}




\begin{tabular}{|c|c|c|c|c|c|c|}
\hline Date & Latitude & Longitude & Location & No. & Source & Remarks \\
\hline 1971 & $40-45^{\circ} \mathrm{N}$ & $160-170^{\circ} \mathrm{E}$ & NW Pacific & 1 & Wada, 1975 & Japanese scoutboat sighting \\
\hline 1971 & $45-50^{\circ} \mathrm{N}$ & $170-180^{\circ} \mathrm{E}$ & South of Aleutians & 4 & Wada, 1975 & Japanese scoutboat sighting \\
\hline 1972 & $40-45^{\circ} \mathrm{N}$ & $160-170^{\circ} \mathrm{E}$ & NW Pacific & 3 & Wada, 1975 & Japanese scoutboat sighting \\
\hline 1972 & $45-50^{\circ} \mathrm{N}$ & $160-170^{\circ} \mathrm{E}$ & NW Pacific, southeast of Kamchatka & 3 & Wada, 1975 & Japanese scoutboat sighting \\
\hline 1972 & $35-40^{\circ} \mathrm{N}$ & $170-180^{\circ} \mathrm{E}$ & NW Pacific & 1 & Wada, 1975 & Japanese scoutboat sighting \\
\hline 1972 & $45-50^{\circ} \mathrm{N}$ & $170-180^{\circ} \mathrm{E}$ & South of Aleutians & 3 & Wada, 1975 & Japanese scoutboat sighting \\
\hline Sep. 1973 & & & Eastern Okhotsk Sea & 16 & Berzin and Vladimirov, 1989 & Seen by factory ship Vladivostok \\
\hline Aug.-Oct. 1974 & & & $\begin{array}{l}\text { Central and northeastern Okhotsk Sea, NE of Kashevarov } \\
\text { Bank }\end{array}$ & $40-45$ & Kuz’min and Berzin, 1975 & Seen by RV Tamango \\
\hline 1974 & $30-50^{\circ} \mathrm{N}$ & $160-180^{\circ} \mathrm{E}$ & West central North Pacific & 4 & Anon., 1976 & Japanese scoutboat sighting \\
\hline 1974 & & & Coast of Japan & 32 & Anon., 1976 & No further details \\
\hline 1975 & $30-50^{\circ} \mathrm{N}$ & $160-180^{\circ} \mathrm{E}$ & West central North Pacific & 4 & Anon., 1977 & Japanese catcherboat sightings \\
\hline 1976 & $40-45^{\circ} \mathrm{N}$ & $170-175^{\circ} \mathrm{E}$ & South of central Aleutians & 1 & Wada, 1978 & Japanese sighting cruise \\
\hline Summer 1981 & $30-40^{\circ} \mathrm{N}$ & $140-150^{\circ} \mathrm{E}$ & Coastal Japan & 3 & Anon., 1983 & Japanese catcherboat sightings \\
\hline 1983 & $30-40^{\circ} \mathrm{N}$ & $140-160^{\circ} \mathrm{E}$ & Northwestern Pacific east of Japan & 2 & Anon., 1985 & Japanese sighting cruise \\
\hline 1983 & $30-40^{\circ} \mathrm{N}$ & $120-140^{\circ} \mathrm{E}$ & Coast of Japan & 2 & Anon., 1985 & Japanese sighting cruise \\
\hline 13 Jan. 1984 & $35^{\circ} 44^{\prime} \mathrm{N}$ & $141^{\circ} 35^{\prime} \mathrm{E}$ & Coast of Japan, off eastern Honshu & 2 & Miyashita and Kato, 1998 & Japanese sighting cruise \\
\hline 21 Jan. 1984 & $33^{\circ} 40^{\prime} \mathrm{N}$ & $138^{\circ} 28^{\prime} \mathrm{E}$ & Coast of Japan, off eastern Honshu & 2 & Miyashita and Kato, 1998 & Japanese sighting cruise \\
\hline 1985 & $30-40^{\circ} \mathrm{N}$ & $140-150^{\circ} \mathrm{E}$ & Coast of Japan & 1 & Anon., 1987 & Japanese catcherboat sighting \\
\hline Jun. 1986 & $50^{\circ} \mathrm{N}$ & $156^{\circ} \mathrm{E}$ & $10-11 \mathrm{~m}$ east of Paramushir I. & 2 & Blokhin, 1988 & One $13-14 \mathrm{~m}$, one $10-11 \mathrm{~m}$ \\
\hline 18 Aug. 1989 & $53^{\circ} 41^{\prime} \mathrm{N}$ & $146^{\circ} 12^{\prime} \mathrm{E}$ & Okhotsk Sea, E of Sakhalin I. & 1 & Miyashita and Kato, 1998 & Japanese sighting cruise; individual resighted 17 Sep. 1992 \\
\hline 8 Apr. 1990 & $27^{\circ} 09^{\prime} \mathrm{N}$ & $142^{\circ} 10^{\prime} \mathrm{E}$ & Chichi-jima I. (Bonin Is), Japan & 1 & Mori et al., 1998 & \\
\hline 11 Aug. 1990 & $50^{\circ} 10^{\prime} \mathrm{N}$ & $154^{\circ} 27^{\prime} \mathrm{E}$ & Okhotsk Sea, W of Paramushir I. & 1 & Miyashita and Kato, 1998 & Japanese sighting cruise \\
\hline 6 Sep. 1990 & $54^{\circ} 28^{\prime} \mathrm{N}$ & $151^{\circ} 08^{\prime} \mathrm{E}$ & Okhotsk Sea & 4 & Miyashita and Kato, 1998 & Japanese sighting cruise \\
\hline 6 Sep. 1990 & $54^{\circ} 29^{\prime} \mathrm{N}$ & $152^{\circ} 07^{\prime} \mathrm{E}$ & Okhotsk Sea & 1 & Miyashita and Kato, 1998 & Japanese sighting cruise \\
\hline 7 Aug. 1992 & $51^{\circ} 17^{\prime} \mathrm{N}$ & $148^{\circ} 38^{\prime} \mathrm{E}$ & Central Okhotsk Sea & 1 & Miyashita and Kato, 1998 & Japanese sighting cruise \\
\hline 26 Aug. 1992 & $51^{\circ} 52^{\prime} \mathrm{N}$ & $155^{\circ} 10^{\prime} \mathrm{E}$ & Okhotsk Sea, off western Kamchatka & 1 & Miyashita and Kato, 1998 & Japanese sighting cruise \\
\hline 26 Aug. 1992 & $51^{\circ} 50^{\prime} \mathrm{N}$ & $155^{\circ} 20^{\prime} \mathrm{E}$ & Okhotsk Sea, off western Kamchatka & 5 & Miyashita and Kato, 1998 & Japanese sighting cruise \\
\hline 26 Aug. 1992 & $51^{\circ} 46^{\prime} \mathrm{N}$ & $155^{\circ} 19^{\prime} \mathrm{E}$ & Okhotsk Sea, off western Kamchatka & 1 & Miyashita and Kato, 1998 & Japanese sighting cruise \\
\hline 26 Aug. 1992 & $51^{\circ} 47^{\prime} \mathrm{N}$ & $155^{\circ} 23^{\prime} \mathrm{E}$ & Okhotsk Sea, off western Kamchatka & 4 & Miyashita and Kato, 1998 & Japanese sighting cruise \\
\hline 26 Aug. 1992 & $51^{\circ} 47^{\prime} \mathrm{N}$ & $155^{\circ} 22^{\prime} \mathrm{E}$ & Okhotsk Sea, off western Kamchatka & 1 & Miyashita and Kato, 1998 & Japanese sighting cruise; animal's tail stock entangled in heavyrope \\
\hline 27 Aug. 1992 & $51^{\circ} 26^{\prime} \mathrm{N}$ & $155^{\circ} 59^{\prime} \mathrm{E}$ & Okhotsk Sea, off western Kamchatka & 2 & Miyashita and Kato, 1998 & Japanese sighting cruise \\
\hline 27 Aug. 1992 & $51^{\circ} 20^{\prime} \mathrm{N}$ & $156^{\circ} 02^{\prime} \mathrm{E}$ & Okhotsk Sea, off western Kamchatka & 1 & Miyashita and Kato, 1998 & Japanese sighting cruise \\
\hline 27 Aug. 1992 & $51^{\circ} 21^{\prime} \mathrm{N}$ & $156^{\circ} 04^{\prime} \mathrm{E}$ & Okhotsk Sea, off western Kamchatka & 2 & Miyashita and Kato, 1998 & Japanese sighting cruise \\
\hline 27 Aug. 1992 & $51^{\circ} 22^{\prime} \mathrm{N}$ & $156^{\circ} 07^{\prime} \mathrm{E}$ & Okhotsk Sea, off western Kamchatka & 1 & Miyashita and Kato, 1998 & Japanese sighting cruise \\
\hline 27 Aug. 1992 & $51^{\circ} 16^{\prime} \mathrm{N}$ & $155^{\circ} 52^{\prime} \mathrm{E}$ & Okhotsk Sea, off western Kamchatka & 1 & Miyashita and Kato, 1998 & Japanese sighting cruise \\
\hline 6 Sep. 1992 & $55^{\circ} 48^{\prime} \mathrm{N}$ & $147^{\circ} 33^{\prime} \mathrm{E}$ & Okhotsk Sea, northeast of Sakhalin I. & 2 & Miyashita and Kato, 1998 & Japanese sighting cruise \\
\hline 9 Sep. 1992 & $54^{\circ} 07^{\prime} \mathrm{N}$ & $152^{\circ} 14^{\prime} \mathrm{E}$ & Central Okhotsk Sea & 2 & Miyashita and Kato, 1998 & Japanese sighting cruise \\
\hline 15 Sep. 1992 & $53^{\circ} 45^{\prime} \mathrm{N}$ & $145^{\circ} 07^{\prime} \mathrm{E}$ & Okhotsk Sea, northeast of Sakhalin I. & 1 & Miyashita and Kato, 1998 & Japanese sighting cruise \\
\hline 15 Sep. 1992 & $53^{\circ} 42^{\prime} \mathrm{N}$ & $145^{\circ} 12^{\prime} \mathrm{E}$ & Okhotsk Sea, northeast of Sakhalin I. & 1 & Miyashita and Kato, 1998 & Japanese sighting cruise \\
\hline 16 Sep. 1992 & $53^{\circ} 12^{\prime} \mathrm{N}$ & $146^{\circ} 31^{\prime} \mathrm{E}$ & Okhotsk Sea, east of Sakhalin I. & 2 & Miyashita and Kato, 1998 & Japanese sighting cruise \\
\hline 17 Sep. 1992 & $51^{\circ} 32^{\prime} \mathrm{N}$ & $144^{\circ} 47^{\prime} \mathrm{E}$ & Okhotsk Sea, east of Sakhalin I. & 1 & Miyashita and Kato, 1998 & Japanese sighting cruise; individual previously observed 18 Aug. 1989 \\
\hline 18 Sep. 1992 & $50^{\circ} 21^{\prime} \mathrm{N}$ & $145^{\circ} 00^{\prime} \mathrm{E}$ & Okhotsk Sea, east of Sakhalin I. & 1 & Miyashita and Kato, 1998 & Japanese sighting cruise \\
\hline 18 Sep. 1992 & $50^{\circ} 18^{\prime} \mathrm{N}$ & $145^{\circ} 01^{\prime} \mathrm{E}$ & Okhotsk Sea, east of Sakhalin I. & 2 & Miyashita and Kato, 1998 & Japanese sighting cruise \\
\hline 18 Sep. 1992 & $50^{\circ} 19^{\prime} \mathrm{N}$ & $145^{\circ} 11^{\prime} \mathrm{E}$ & Okhotsk Sea, east of Sakhalin I. & 1 & Miyashita and Kato, 1998 & Japanese sighting cruise \\
\hline 18 Sep. 1992 & $50^{\circ} 17^{\prime} \mathrm{N}$ & $145^{\circ} 12^{\prime} \mathrm{E}$ & Okhotsk Sea, east of Sakhalin I. & 1 & Miyashita and Kato, 1998 & Japanese sighting cruise \\
\hline
\end{tabular}




\begin{tabular}{|c|c|c|c|c|c|c|}
\hline Date & Latitude & Longitude & Location & No. & Source & Remarks \\
\hline 12 Apr. 1993 & $27^{\circ} 06^{\prime} \mathrm{N}$ & $142^{\circ} 10^{\prime} \mathrm{E}$ & Chichi-jima I. (Bonin I.), Japan & 2 & Mori et al., 1998 & With humpback whales \\
\hline 15 Apr. 1993 & $27^{\circ} 02^{\prime} \mathrm{N}$ & $142^{\circ} 10^{\prime} \mathrm{E}$ & Chichi-jima I. (Bonin I.), Japan & 1 & Mori et al., 1998 & One animal from pair on $12 \mathrm{Apr}$. \\
\hline 12 Aug. 1994 & $42^{\circ} 10^{\prime} \mathrm{N}$ & $142^{\circ} 08^{\prime} \mathrm{E}$ & Northwestern Pacific & 2 & Fujise et al., 1995 & Cow $\left(51^{\prime}\right)$ and calf $\left(27^{\prime}\right)$ \\
\hline 21 Jun. 1995 & $47^{\circ} 28^{\prime} \mathrm{N}$ & $163^{\circ} 19^{\prime} \mathrm{E}$ & Northwestern Pacific & 1 & Fujise et al., 1996 & \\
\hline 9 Aug. 1995 & $45^{\circ} 00^{\prime} \mathrm{N}$ & $165^{\circ} 34^{\prime} \mathrm{E}$ & Northwestern Pacific & 1 & Fujise et al., 1996 & \\
\hline 13 Mar. 1996 & $27^{\circ} 04^{\prime} \mathrm{N}$ & $142^{\circ} 08^{\prime} \mathrm{E}$ & Chichi-jima I. (Bonin Is), Japan & 1 & Mori et al., 1998 & Estimated length $10 \mathrm{~m}$; with humpback whales \\
\hline 1 May 1996 & $34^{\circ} 45^{\prime} \mathrm{N}$ & $138^{\circ} 44^{\prime} \mathrm{E}$ & Off coast of Izu, Japan & 1 & T. Kasuya, unpub. & Estimated length $10 \mathrm{~m}$ \\
\hline 8 Aug. 1996 & $45^{\circ} 52^{\prime} \mathrm{N}$ & $159^{\circ} 38^{\prime} \mathrm{E}$ & Northwestern North Pacific & 1 & NRIFS data & Japanese sighting cruise \\
\hline 12 Apr. 1997 & & & Yakiuchi-wan, Amami-Oshima & 1 & S. Uchida, unpub. & Japanese sighting cruise \\
\hline 15 May 1997 & $51^{\circ} 06^{\prime} \mathrm{N}$ & $166^{\circ} 08^{\prime} \mathrm{E}$ & East of Japan (sub-area 9) & 1 & JARPN 1997 cruise report & Japanese sighting cruise \\
\hline 26 May 1997 & $46^{\circ} 10^{\prime} \mathrm{N}$ & $162^{\circ} 30^{\prime} \mathrm{E}$ & Northwestern Pacific & 2 & Ishikawa et al., 1997 & Japanese sighting cruise \\
\hline Apr--Jun. 1998 & $39-41^{\circ} \mathrm{N}$ & $145-150^{\circ} \mathrm{E}$ & East of Japan (sub-area 7) & 3 & Zenitani et al., 1999 & Japanese sighting cruise \\
\hline 9 Jun. 1999 & & & 10-15 miles off Taiji, Wakayama, Japan & 1 & K. Mori, pers. comm. & Observed by M. Hohana, Taiji, Japan \\
\hline 10 Sep. 1999 & $50^{\circ} 08^{\prime} \mathrm{N}$ & $145^{\circ} 31^{\prime} \mathrm{E}$ & Okhotsk Sea, east of Sakhalin Island & 2 & Miyashita et al., 2000; NRIFS data & Japanese sighting cruise \\
\hline 15 Sep. 1999 & $53^{\circ} 01^{\prime} \mathrm{N}$ & $153^{\circ} 59^{\prime} \mathrm{E}$ & Okhotsk Sea, off western Kamchatka & 2 & Miyashita et al., 2000; NRIFS data & Japanese sighting cruise \\
\hline 16 Sep. 1999 & $52^{\circ} 13^{\prime} \mathrm{N}$ & $154^{\circ} 27^{\prime} \mathrm{E}$ & Okhotsk Sea, off western Kamchatka & 3 & Miyashita et al., 2000; NRIFS data & Japanese sighting cruise \\
\hline 16 Sep. 1999 & $52^{\circ} 04^{\prime} \mathrm{N}$ & $154^{\circ} 06^{\prime} \mathrm{E}$ & Okhotsk Sea, off western Kamchatka & 1 & Miyashita et al., 2000; NRIFS data & Japanese sighting cruise \\
\hline 16 Sep. 1999 & $51^{\circ} 46^{\prime} \mathrm{N}$ & $153^{\circ} 30^{\prime} \mathrm{E}$ & Okhotsk Sea, off western Kamchatka & 2 & Miyashita et al., 2000; NRIFS data & Japanese sighting cruise \\
\hline 17 Sep. 1999 & $51^{\circ} 24^{\prime} \mathrm{N}$ & $152^{\circ} 46^{\prime} \mathrm{E}$ & Okhotsk Sea, off western Kamchatka & 1 & Miyashita et al., 2000; NRIFS data & Japanese sighting cruise \\
\hline
\end{tabular}

Table 2.2

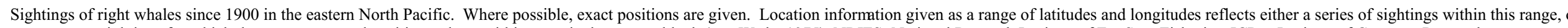
sightings for which the source reported position only to within a particular survey block (e.g. Wada, 1975). NRIFS=National Research Institute of Far Seas Fisheries; ICR = Institute of Cetacean Research

\begin{tabular}{|c|c|c|c|c|c|c|}
\hline Date & Latitude & Longitude & Location & No. & Source & Remarks \\
\hline 27 Jun. 1928 & & & 20m SE of Rootok I. Alaska & 2 & Reeves et al., 1985 (table 8) & Chased for $1 \mathrm{hr}$ by catcherboat Paterson \\
\hline 4 Aug. 1928 & & & Off Akutan, Alaska & 1 & Reeves et al., 1985 (p.455) & Struck and lost during whaling by catcherboat Unimak \\
\hline 3 Sep. 1929 & & & 40m off Rootok I. Alaska & 1 & Reeves et al., 1985 (table 8) & Chased for $6 \mathrm{hr} 20 \mathrm{~min}$ by catcherboat Unimak \\
\hline 11 May 1937 & & & $\mathrm{Ca} 20 \mathrm{~m}$ SE of Twohead I. (Kodiak) & 2 & Reeves et al., 1985 (table 8) & Chased by catcherboat Moran \\
\hline 14 May 1937 & & & $\mathrm{Ca} 1 \mathrm{~m} \mathrm{SE}$ of Twohead I. (Kodiak) & 1 & Reeves et al., 1985 (table 8) & Chased for $1 \mathrm{hr}$ by catcherboat Tanginak \\
\hline 20 Jun. 1937 & & & SE of Unalaska I. Alaska & 1 & Reeves et al., 1985 (table 8) & Chased by catcherboat Paterson \\
\hline 24 Aug. 1937 & & & $28 \mathrm{~m} \mathrm{SE}$ of Rootok I. Alaska & 1 & Reeves et al., 1985 (table 8) & Seen by catcherboat Kodiak \\
\hline 17 Aug. 1939 & & & Area of Akutan Pass & 1 & Reeves et al., 1985 (table 8) & Seen by catcherboat Kodiak \\
\hline \multirow[t]{2}{*}{ Jun. 1941-1957 } & & & Central and eastern Aleutians (Pacific side) & 19 & Omura, 1958 & Japanese catcherboat sightings \\
\hline & & & Eastern Aleutians, in SE Bering Sea & 2 & & \\
\hline Jul. 1941-1957 & & & E Aleutians (both sides), and SE Bering Sea & 92 & Omura, 1958 & Japanese catcherboat sightings \\
\hline Sep. 1941-1957 & $53^{\circ} \mathrm{N}$ & $168^{\circ} \mathrm{W}$ & E Aleutians (Pacific side) & 3 & Omura, 1958 & Japanese catcherboat sightings \\
\hline 31 Mar. 1955 & $32^{\circ} 50^{\prime} \mathrm{N}$ & $117^{\circ} 30^{\prime} \mathrm{W}$ & La Jolla, California & 1 & Gilmore, 1956 & \\
\hline 1955-1958 & $40-50^{\circ} \mathrm{N}$ & $170-180^{\circ} \mathrm{W}$ & South of Aleutians & 4 & Slijper et al., 1964 & Opportunistic sightings, Dutch vessels \\
\hline 1958 & & & 'East of longitude 180 degrees' & 12 & ICR data & Japanese catcherboat sightings \\
\hline Post-1958? & $50^{\circ} \mathrm{N}$ & $145^{\circ} \mathrm{W}$ & Northwest Ground & 2 & Pike and McAskie, 1969 & Weather ship sightings \\
\hline
\end{tabular}




\begin{tabular}{|c|c|c|c|c|c|c|}
\hline Date & Latitude & Longitude & Location & No. & Source & Remarks \\
\hline 1958-1964 & & & $\begin{array}{l}\text { Eastern Aleutians (both sides) } \\
\text { SE Bering Sea } \\
\text { NE Pacific } \\
\text { Northwest Ground }\end{array}$ & $\begin{array}{c}65-120 \\
63-114 \\
2-6 \\
88-189\end{array}$ & Berzin and Rovnin, 1966 & $\begin{array}{l}\text { Soviet sighting surveys. Shown (fig. 6) as plots of either ' } 1-3 \text { ' or ' } 15-20 \text { ' whales. Includes } \\
200 \text { reported in } 1963 \text { by Berzin and Doroshenko (1982), but locations in two papers } \\
\text { conflict }\end{array}$ \\
\hline 8 Apr. 1959 & $45^{\circ} 55^{\prime} \mathrm{N}$ & $125^{\circ} 25^{\prime} \mathrm{W}$ & $80 \mathrm{~m} \mathrm{~W}$ of Tillamook Hd, Washington & 3 & Fiscus and Niggol, 1965 & $\begin{array}{l}\text { Seen during fur seal research cruise; probably same group resighted near this position two } \\
\text { days later }\end{array}$ \\
\hline 13-15 May 1959 & $37^{\circ} 25^{\prime} \mathrm{N}$ & $122^{\circ} 48^{\prime} \mathrm{W}$ & $16 \mathrm{~m} \mathrm{SW}$ of Pt Montara, California & 1 & Rice and Fiscus, 1968 & 40 feet \\
\hline 6 Jul. 1959 & $57^{\circ} 23^{\prime} \mathrm{N}$ & $174^{\circ} 01^{\prime} \mathrm{W}$ & Central Bering Sea & 1 & Japan Whaling Association Data & Japanese catcherboat sighting \\
\hline 7 Jul. 1959 & $56^{\circ} 02^{\prime} \mathrm{N}$ & $171^{\circ} 28^{\prime} \mathrm{W}$ & SE Bering Sea & 5 & Japan Whaling Association Data & Japanese catcherboat sightings \\
\hline 8 Jul. 1959 & $54^{\circ} 25 ’ \mathrm{~N}$ & $167^{\circ} 53^{\prime} \mathrm{W}$ & Eastern Aleutians & 2 & Japan Whaling Association Data & Japanese catcherboat sightings \\
\hline 14 Jul. 1959 & $54^{\circ} 57^{\prime} \mathrm{N}$ & $167^{\circ} 11^{\prime} \mathrm{W}$ & Eastern Aleutians & 4 & Japan Whaling Association Data & Japanese catcherboat sightings \\
\hline 15 Jul. 1959 & $54^{\circ} 27^{\prime} \mathrm{N}$ & $168^{\circ} 10^{\prime} \mathrm{W}$ & Eastern Aleutians & 5 & Japan Whaling Association Data & Japanese catcherboat sightings \\
\hline 11 Jun. 1960 & $52^{\circ} 34^{\prime} \mathrm{N}$ & $175^{\circ} 16^{\prime} \mathrm{W}$ & Central Aleutians & 1 & Japan Whaling Association Data & Japanese catcherboat sighting \\
\hline 13 Jun. 1960 & $54^{\circ} 25^{\prime} \mathrm{N}$ & $169^{\circ} 55^{\prime} \mathrm{W}$ & Eastern Aleutians & 5 & Japan Whaling Association Data & Japanese catcherboat sightings \\
\hline 15 Jun. 1960 & $55^{\circ} 45^{\prime} \mathrm{N}$ & $168^{\circ} 48^{\prime} \mathrm{W}$ & Eastern Aleutians & 10 & Japan Whaling Association Data & Japanese catcherboat sightings \\
\hline 19 Jun. 1960 & $54^{\circ} 30^{\prime} \mathrm{N}$ & $168^{\circ} 22^{\prime} \mathrm{W}$ & Eastern Aleutians & 3 & Japan Whaling Association Data & Japanese catcherboat sightings \\
\hline 21 Jun. 1960 & $55^{\circ} 31^{\prime} \mathrm{N}$ & $169^{\circ} 41^{\prime} \mathrm{W}$ & SE Bering Sea & 1 & Japan Whaling Association Data & Japanese catcherboat sighting \\
\hline 22 Jun. 1960 & $55^{\circ} 45^{\prime} \mathrm{N}$ & $170^{\circ} 40^{\prime} \mathrm{W}$ & SE Bering Sea & 5 & Japan Whaling Association Data & \\
\hline & & & & & & Japanese catcherboat sightings \\
\hline 23 Jun. 1960 & $56^{\circ} 12^{\prime} \mathrm{N}$ & $170^{\circ} 03^{\prime} \mathrm{W}$ & SE Bering Sea & 3 & Japan Whaling Association Data & Japanese catcherboat sightings \\
\hline 24 Jun. 1960 & $56^{\circ} 10^{\prime} \mathrm{N}$ & $170^{\circ} 39^{\prime} \mathrm{W}$ & SE Bering Sea & 6 & Japan Whaling Association Data & Japanese catcherboat sightings \\
\hline 25 Jun. 1960 & $54^{\circ} 48^{\prime} \mathrm{N}$ & $167^{\circ} 51^{\prime} \mathrm{W}$ & Eastern Aleutians & 4 & Japan Whaling Association Data & Japanese catcherboat sightings \\
\hline 26 Jun. 1960 & $54^{\circ} 38^{\prime} \mathrm{N}$ & $167^{\circ} 59^{\prime} \mathrm{W}$ & Eastern Aleutians & 1 & Japan Whaling Association Data & Japanese catcherboat sighting \\
\hline 28 Jun. 1960 & $53^{\circ} 49^{\prime} \mathrm{N}$ & $170^{\circ} 17^{\prime} \mathrm{W}$ & Eastern Aleutians & 1 & Japan Whaling Association Data & Japanese catcherboat sighting \\
\hline 30 Jun. 1960 & $55^{\circ} 51^{\prime} \mathrm{N}$ & $171^{\circ} 18^{\prime} \mathrm{W}$ & SE Bering Sea & 3 & Japan Whaling Association Data & Japanese catcherboat sightings \\
\hline 1 Jul. 1960 & $56^{\circ} 19^{\prime} \mathrm{N}$ & $171^{\circ} 21^{\prime} \mathrm{W}$ & SE Bering Sea & 1 & Japan Whaling Association Data & Japanese catcherboat sighting \\
\hline 2 Jul. 1960 & $55^{\circ} 48^{\prime} \mathrm{N}$ & $171^{\circ} 19^{\prime} \mathrm{W}$ & SE Bering Sea & 5 & Japan Whaling Association Data & Japanese catcherboat sightings \\
\hline 6 Jul. 1960 & $50^{\circ} 44^{\prime} \mathrm{N}$ & $167^{\circ} 27^{\prime} \mathrm{W}$ & $\mathrm{S}$ of eastern Aleutians & 2 & Japan Whaling Association Data & Japanese catcherboat sightings \\
\hline 9 Jul. 1960 & $54^{\circ} 05^{\prime} \mathrm{N}$ & $160^{\circ} 20^{\prime} \mathrm{W}$ & S of Alaska Peninsula & 2 & Japan Whaling Association Data & Japanese catcherboat sightings \\
\hline 10 Jul. 1960 & $54^{\circ} 49^{\prime} \mathrm{N}$ & $158^{\circ} 06^{\prime} \mathrm{W}$ & S of Alaska Peninsula & 2 & Japan Whaling Association Data & Japanese catcherboat sightings \\
\hline 12 Jul. 1960 & $54^{\circ} 34^{\prime} \mathrm{N}$ & $155^{\circ} 11^{\prime} \mathrm{W}$ & Northwest Ground & 1 & Japan Whaling Association Data & Japanese catcherboat sighting \\
\hline 15 Jul. 1960 & $53^{\circ} 39^{\prime} \mathrm{N}$ & $164^{\circ} 04^{\prime} \mathrm{W}$ & S of Alaska Peninsula & 2 & Japan Whaling Association Data & Japanese catcherboat sightings \\
\hline 16 Jul. 1960 & $52^{\circ} 51^{\prime} \mathrm{N}$ & $165^{\circ} 53^{\prime} \mathrm{W}$ & $\mathrm{S}$ of eastern Aleutians & 1 & Japan Whaling Association Data & Japanese catcherboat sighting \\
\hline 20 Jul. 1960 & $50^{\circ} 05^{\prime} \mathrm{N}$ & $174^{\circ} 21^{\prime} \mathrm{W}$ & Central Aleutians & 1 & Japan Whaling Association Data & Japanese catcherboat sighting \\
\hline 21 Jul. 1960 & $52^{\circ} 47^{\prime} \mathrm{N}$ & $171^{\circ} 40^{\prime} \mathrm{W}$ & Eastern Aleutians & 4 & Japan Whaling Association Data & Japanese catcherboat sightings \\
\hline 22 Jul. 1960 & $54^{\circ} 33^{\prime} \mathrm{N}$ & $169^{\circ} 39^{\prime} \mathrm{W}$ & SE Bering Sea & 13 & Japan Whaling Association Data & Japanese catcherboat sightings \\
\hline 9 Aug. 1960 & $58^{\circ} 36^{\prime} \mathrm{N}$ & $174^{\circ} 47^{\prime} \mathrm{W}$ & Central Bering Sea & 2 & Japan Whaling Association Data & Japanese catcherboat sightings \\
\hline 10 Aug. 1960 & $58^{\circ} 16^{\prime} \mathrm{N}$ & $174^{\circ} 42^{\prime} \mathrm{W}$ & Central Bering Sea & 2 & Japan Whaling Association Data & Japanese catcherboat sightings \\
\hline 12 Aug. 1960 & $56^{\circ} 41^{\prime} \mathrm{N}$ & $173^{\circ} 25^{\prime} \mathrm{W}$ & SE Bering Sea & 1 & Japan Whaling Association Data & Japanese catcherboat sighting \\
\hline 14 Aug. 1960 & $53^{\circ} 42^{\prime} \mathrm{N}$ & $170^{\circ} 35^{\prime} \mathrm{W}$ & SE Bering Sea & 4 & Japan Whaling Association Data & Japanese catcherboat sightings \\
\hline 21 Jun. 1961 & $56^{\circ} 11^{\prime} \mathrm{N}$ & $172^{\circ} 13^{\prime} \mathrm{W}$ & SE Bering Sea & 2 & Japan Whaling Association Data & Japanese catcherboat sightings \\
\hline 6 Jul. 1961 & $55^{\circ} 01^{\prime} \mathrm{N}$ & $167^{\circ} 48^{\prime} \mathrm{W}$ & Eastern Aleutians & 2 & Japan Whaling Association Data & Japanese catcherboat sightings \\
\hline 8 Aug. 1961 & $58^{\circ} 04^{\prime} \mathrm{N}$ & $149^{\circ} 24^{\prime} \mathrm{W}$ & Gulf of Alaska & 1 & Japan Whaling Association Data & Japanese catcherboat sighting \\
\hline 21 Aug. 1961 & $56^{\circ} 32^{\prime} \mathrm{N}$ & $152^{\circ} 14^{\prime} \mathrm{W}$ & Kodiak Island & 4 & Japan Whaling Association Data & Japanese catcherboat sightings \\
\hline 22 Aug. 1961 & $56^{\circ} 03^{\prime} \mathrm{N}$ & $153^{\circ} 00^{\prime} \mathrm{W}$ & Kodiak Island & 3 & Japan Whaling Association Data & Japanese catcherboat sightings \\
\hline 29 Aug. 1961 & $52^{\circ} 44^{\prime} \mathrm{N}$ & $173^{\circ} 58^{\prime} \mathrm{W}$ & Central Aleutians & 2 & Japan Whaling Association Data & Japanese catcherboat sightings \\
\hline
\end{tabular}




\begin{tabular}{|c|c|c|c|c|c|c|}
\hline Date & Latitude & Longitude & Location & No. & Source & Remarks \\
\hline 1961 & & & Gulf of Alaska & 1 & Omura and Ohsumi, 1964 & Marked; may be duplicate of 8 Aug. sighting above \\
\hline 13 Jul. 1962 & $50^{\circ} 22^{\prime} \mathrm{N}$ & $165^{\circ} 32^{\prime} \mathrm{W}$ & Northeastern North Pacific & 3 & NRIFS data & Japanese catcherboat sighings \\
\hline 16 Jul. 1962 & $52^{\circ} 07^{\prime} \mathrm{N}$ & $169^{\circ} 44^{\prime} \mathrm{W}$ & Eastern Aleutians, Pacific side & 1 & NRIFS data & Japanese catcherboat sighting \\
\hline 1962 & & & Eastern Bering Sea & 1 & Omura and Ohsumi, 1964 & Marked \\
\hline 1962 & & & 'East of longitude 180 degrees’ & 4 & Japan Whaling Association Data & One may be duplicate of sighting on previous line \\
\hline 11 Apr. 1963 & $37^{\circ} 08^{\prime} \mathrm{N}$ & $123^{\circ} 05^{\prime} \mathrm{W}$ & 33m W of Pigeon Pt., California & 1 & Rice and Fiscus, 1968 & $<30$ feet \\
\hline 10 May 1963 & $37^{\circ} 20^{\prime} \mathrm{N}$ & $123^{\circ} 10^{\prime} \mathrm{W}$ & 24m SSW Farallon Is., California & 1 & Rice and Fiscus, 1968 & 45 feet \\
\hline 1963 & $51^{\circ} \mathrm{N}$ & $145^{\circ} \mathrm{W}$ & Northwest Ground (but see Remarks) & 200 & Berzin and Doroshenko, 1982 & $\begin{array}{l}\text { Sighting by Soviet research vessels. Berzin and Rovnin (1966) say } 200 \text { in all eastern North } \\
\text { Pacific in 1963, and their plot (fig. 6) shows no large sightings near this location }\end{array}$ \\
\hline Jan. 1964 & $40^{\circ} \mathrm{N}$ & $157^{\circ} \mathrm{W}$ & NE Pacific & ? & Berzin and Doroshenko, 1982 & ‘Right whales’ seen by Soviet RV \\
\hline 11 Mar. 1965 & $26^{\circ} 39^{\prime} \mathrm{N}$ & $113^{\circ} 40^{\prime} \mathrm{W}$ & 6-7m SW of Pta Abreojos, Baja California & 2 & Rice and Fiscus, 1968 & Both approx. 50 feet \\
\hline 1965 & $50-55^{\circ} \mathrm{N}$ & $160-170^{\circ} \mathrm{W}$ & Aleutians, Pacific side & 1 & Wada, 1975 & Japanese scoutboat sighting \\
\hline 1965 & $55-60^{\circ} \mathrm{N}$ & $140-150^{\circ} \mathrm{W}$ & Gulf of Alaska & 1 & Wada, 1975 & Japanese scoutboat sighting \\
\hline 1965 & $45-50^{\circ} \mathrm{N}$ & $170-180^{\circ} \mathrm{W}$ & S of Aleutians & 1 & Wada, 1975 & Japanese scoutboat sighting \\
\hline 1966 & $50-55^{\circ} \mathrm{N}$ & $170-180^{\circ} \mathrm{W}$ & Aleutians, Pacific side & 1 & Wada, 1975 & Japanese scoutboat sightings \\
\hline 1966 & $50-55^{\circ} \mathrm{N}$ & $150-160^{\circ} \mathrm{W}$ & S of Alaska Peninsula & 3 & Wada, 1975 & Japanese scoutboat sighting \\
\hline 1966 & $55-60^{\circ} \mathrm{N}$ & $140-150^{\circ} \mathrm{W}$ & Gulf of Alaska & 1 & Wada, 1975 & Japanese scoutboat sightings \\
\hline 17 Jan. 1967 & $48^{\circ} 20^{\prime} \mathrm{N}$ & $125^{\circ} 06^{\prime} \mathrm{W}$ & 15m WSW Cape Flattery, Washington & 3 & Rice and Fiscus, 1968 & \\
\hline 1967 & $55-60^{\circ} \mathrm{N}$ & $160-170^{\circ} \mathrm{W}$ & Eastern Bering Sea & 1 & Wada, 1975 & Japanese scoutboat sighting \\
\hline 1967 & $50-55^{\circ} \mathrm{N}$ & $150-160^{\circ} \mathrm{W}$ & S of Alaska Peninsula & 1 & Wada, 1975 & Japanese scoutboat sighting \\
\hline 1969 & $50-55^{\circ} \mathrm{N}$ & $160-170^{\circ} \mathrm{W}$ & Aleutians, Pacific side & 1 & Wada, 1975 & Japanese scoutboat sighting \\
\hline 1969 & $45-50^{\circ} \mathrm{N}$ & $170-180^{\circ} \mathrm{W}$ & S of Aleutians & 1 & Wada, 1975 & Japanese scoutboat sighting \\
\hline 1970 & $50-55^{\circ} \mathrm{N}$ & $130-140^{\circ} \mathrm{W}$ & W of Queen Charlotte Is, British Columbia & 2 & Wada, 1975 & Japanese scoutboat sightings \\
\hline 1973 & $50-55^{\circ} \mathrm{N}$ & $160-170^{\circ} \mathrm{W}$ & Aleutians, Pacific side & 1 & Wada, 1975 & Japanese scoutboat sighting \\
\hline 1973 & $45-50^{\circ} \mathrm{N}$ & $140-150^{\circ} \mathrm{W}$ & NE Pacific, S of Gulf of Alaska & 1 & Wada, 1975 & Japanese scoutboat sighting \\
\hline 13 Sep. 1974 & $39^{\circ} 35^{\circ} \mathrm{N}$ & $124,45^{\circ} \mathrm{W}$ & $33 \mathrm{~m} \mathrm{~W}$ of Fort Bragg, California & 1 & $\begin{array}{l}\text { NMFS Platform of Opportunity } \\
\text { data; Scarff, 1986a }\end{array}$ & Weather ship sighting \\
\hline 1974 & $40-50^{\circ} \mathrm{N}$ & $140-160^{\circ} \mathrm{W}$ & NE Pacific & 1 & Anon., 1976 & Japanese catcherboat sighting \\
\hline 1975 & $40-45^{\circ} \mathrm{N}$ & $140-150^{\circ} \mathrm{W}$ & NE Pacific, S of Gulf of Alaska & 2 & Wada, 1977 & Japanese sighting cruise \\
\hline 1976 & $50-55^{\circ} \mathrm{N}$ & $155-160^{\circ} \mathrm{W}$ & Kodiak I. & 1 & Wada, 1978 & Japanese sighting cruise \\
\hline 1976 & $45-50^{\circ} \mathrm{N}$ & $150-155^{\circ} \mathrm{W}$ & NE Pacific, $S$ of Alaska Peninsula & 1 & Wada, 1978 & Japanese sighting cruise \\
\hline Summer 1977 & $20-30^{\circ} \mathrm{N}$ & $160-180^{\circ} \mathrm{W}$ & Hawaiian Is region & 2 & Anon., 1979 & Japanese catcherboat sighting \\
\hline 1977 & $45-50^{\circ} \mathrm{N}$ & $150-155^{\circ} \mathrm{W}$ & NE Pacific, $S$ of Alaska Peninsula & 1 & Wada, 1979 & Japanese sighting cruise \\
\hline 1977 & $45-50^{\circ} \mathrm{N}$ & $140-145^{\circ} \mathrm{W}$ & NE Pacific, S of Gulf of Alaska & 1 & Wada, 1979 & Japanese sighting cruise \\
\hline 1977 & $45-50 \mathrm{~N}$ & $135-140^{\circ} \mathrm{W}$ & NE Pacific, S of Gulf of Alaska & 2 & Wada, 1979 & Japanese sighting cruise \\
\hline Summer 1978 & $50-60^{\circ} \mathrm{N}$ & $140-160^{\circ} \mathrm{W}$ & Northwest Ground/Alaska Peninsula region & 2 & Anon., 1980 & Japanese scout boat sightings \\
\hline 25 Mar. 1979 & $20^{\circ} 40^{\prime} \mathrm{N}$ & $156^{\circ} 53^{\prime} \mathrm{W}$ & Off Maui, Hawaii & 1 & $\begin{array}{l}\text { Rowntree et al., } 1980 \\
\text { Herman et al., } 1980\end{array}$ & 15-16m; associated with humpback whales. Resighted 10 Apr. \\
\hline Summer 1979 & $40-45^{\circ} \mathrm{N}$ & $145-150^{\circ} \mathrm{W}$ & NE Pacific & 1 & Wada, 1981 & Japanese sighting cruise \\
\hline 1979 & $40-50^{\circ} \mathrm{N}$ & $140-160^{\circ} \mathrm{W}$ & NE Pacific & 1 & Anon., 1981 & Marked by Japanese \\
\hline 17 Apr. 1981 & $34^{\circ} 07^{\prime} \mathrm{N}$ & $119^{\circ} 18^{\prime} \mathrm{W}$ & Santa Barbara Channel, California & 1 & Woodhouse and Strickley, 1982 & $14 \mathrm{~m}$ \\
\hline 20 Mar. 1982 & $37^{\circ} 30^{\prime} \mathrm{N}$ & $122^{\circ} 30^{\prime} \mathrm{W}$ & Half Moon Bay, near San Francisco & 1 & Johnson, 1982; Scarff, 1986b & $15 \mathrm{~m}$ \\
\hline 26 Jul. 1982 & $60^{\circ} 48^{\prime} \mathrm{N}$ & $175^{\circ} 18^{\prime} \mathrm{W}$ & NW of St Matthew I., Bering Sea & 2 & Brueggeman et al., 1984 & Sighting details provided by G. Joyce \\
\hline 28 Aug. 1983 & $48^{\circ} 33^{\prime} \mathrm{N}$ & $124^{\circ} 39^{\prime} \mathrm{W}$ & Juan de Fuca Strait, British Columbia & 2 & Reeves and Leatherwood, 1985 & \\
\hline 8 Sep. 1985 & $56^{\circ} 54^{\prime} \mathrm{N}$ & $163^{\circ} 56^{\prime} \mathrm{W}$ & SE Bering Sea & 1 & Goddard and Rugh, 1998 & Photographed \\
\hline
\end{tabular}




\begin{tabular}{|c|c|c|c|c|c|c|}
\hline Date & Latitude & Longitude & Location & No. & Source & Remarks \\
\hline 5 Feb. 1988 & $32^{\circ} 50^{\prime} \mathrm{N}$ & $117^{\circ} 30^{\prime} \mathrm{W}$ & La Jolla, California & 1 & Scarff, 1991 & \\
\hline 9 May 1990 & $33^{\circ} 28^{\prime} \mathrm{N}$ & $118^{\circ} 25^{\prime} \mathrm{W}$ & $8 \mathrm{~m} \mathrm{~N}$ of Santa Catalina I., California & 1 & Rae-Dupree and Krikorian, 1990 & Photographed \\
\hline 4 Aug. 1991 & $43^{\circ} 03^{\prime} \mathrm{N}$ & $179^{\circ} 09^{\prime} \mathrm{W}$ & Northeastern North Pacific & 1 & $\begin{array}{l}\text { NRIFS data; NMFS Platform of } \\
\text { Opportunity data }\end{array}$ & Photographed \\
\hline 24 Mar. 1992 & $32^{\circ} 14^{\prime} \mathrm{N}$ & $118^{\circ} 42^{\prime} \mathrm{W}$ & $70 \mathrm{~km} \mathrm{SW}$ of San Clemente I., California & 1 & Carretta et al.., 1994 & Length $12.6 \mathrm{~m}$ \\
\hline 24 May 1992 & $47^{\circ} 17^{\prime} \mathrm{N}$ & $125^{\circ} 11^{\prime} \mathrm{W}$ & $65 \mathrm{~km} \mathrm{~W}$ of Cape Elizabeth, Washington & 1 & Rowlett et al., 1994 & Seen from aerial survey and reidentified $6 \mathrm{hrs}$ later $48 \mathrm{~km} \mathrm{~W}$ of Destruction I. \\
\hline 12 Apr. 1993 & $54^{\circ} 43^{\prime} \mathrm{N}$ & $165^{\circ} 03^{\prime} \mathrm{W}$ & Southeastern Bering Sea & 1 & Goddard and Rugh, 1998 & Sighting K. Vicknair, NMFS Platform of Opportunity data \\
\hline 7 Aug. 1993 & $57^{\circ} 46^{\prime} \mathrm{N}$ & $166^{\circ} 27^{\prime} \mathrm{W}$ & Southeastern Bering Sea & 2 & Goddard and Rugh, 1998 & Sighting D. Morse, NMFS Platform of Opportunity data \\
\hline 3 May 1995 & $35^{\circ} 40^{\prime} \mathrm{N}$ & $121^{\circ} 17^{\prime} \mathrm{W}$ & Off Piedras Blancas, California & 1 & Rowlett, unpub. & Seen from shore \\
\hline 20 Feb. 1996 & $23^{\circ} 02^{\prime} \mathrm{N}$ & $109^{\circ} 30^{\prime} \mathrm{W}$ & 15 miles off Cabo San Lucas, Mexico & 1 & Gendron et al., 1999 & Photographed from aeroplane \\
\hline 2 Apr. 1996 & $20^{\circ} 56^{\prime} \mathrm{N}$ & $156^{\circ} 46^{\prime} \mathrm{W}$ & Off Maui, Hawaii & 1 & Salden and Mickelson, 1999 & \\
\hline 30 Jul. 1996 & $57^{\circ} 36^{\prime} \mathrm{N}$ & $163^{\circ} 21^{\prime} \mathrm{W}$ & Southeastern Bering Sea & 4 & Goddard and Rugh, 1998 & Possibly included calf? \\
\hline 25 Sep. 1996 & $56^{\circ} 48^{\prime} \mathrm{N}$ & $164^{\circ} 24^{\prime} \mathrm{W}$ & Southeastern Bering Sea & $2-4$ & Goddard and Rugh, 1998 & Sighting T. Lewandowski \\
\hline 20 Jul. 1997 & $57^{\circ} 08^{\prime} \mathrm{N}$ & $162^{\circ} 50^{\prime} \mathrm{W}$ & Southeastern Bering Sea & $4-5$ & Tynan, 1998 & Biopsied and photographed \\
\hline 22-23 Oct. 1997 & $56^{\circ} 50^{\prime} \mathrm{N}$ & $164^{\circ} 30^{\prime} \mathrm{W}$ & Southeastern Bering Sea & $1+$ & M. Bomlander, unpub. & Sighting M. Bomlander, NMFS Platform of Opportunity data, photographed \\
\hline 27 Feb. 1998 & $35^{\circ} 44^{\prime} \mathrm{N}$ & $121^{\circ} 30^{\prime} \mathrm{W}$ & Big Sur Coast, California & 1 & Evans, 1998 & Photographed \\
\hline 14-19 Jul. 1998 & $\begin{array}{r}56^{\circ} 46^{\prime}- \\
57^{\circ} 05^{\prime} \mathrm{N}\end{array}$ & $\begin{array}{r}164^{\circ} 15^{\prime}- \\
164^{\circ} 51^{\prime} \mathrm{W}\end{array}$ & Southeastern Bering Sea & 6 & Perryman et al., 1999 & Photographed from aeroplane \\
\hline 14 Jul. 1998 & $57^{\circ} 08^{\prime} \mathrm{N}$ & $151^{\circ} 51^{\prime} \mathrm{W}$ & S of Kodiak I. & 1 & $\mathrm{~K}$. Wynne and J. Waite, unpub. & Photographed \\
\hline 8-17 Jul. 1998 & $\begin{array}{r}56^{\circ} 39^{\prime}- \\
57^{\circ} 07^{\prime} \mathrm{N}\end{array}$ & $\begin{array}{r}163^{\circ} 11^{\prime}- \\
164^{\circ} 18^{\prime} \mathrm{W}\end{array}$ & Southeastern Bering Sea & 5 & LeDuc et al., 2000 & Photographed from aeroplane \\
\hline 15 Jun. 1999 & & & Southeastern Bering Sea & 1 & Tynan, 2001 & \\
\hline 31 Jul. 1999 & $56^{\circ} 53^{\prime} \mathrm{N}$ & $163^{\circ} 33^{\prime} \mathrm{W}$ & Southeastern Bering Sea & 2 & Moore et al., 2001 & Photographed from RV Miller Freeman \\
\hline 29 Oct. 1999 & $56^{\circ} 26^{\prime} \mathrm{N}$ & $164^{\circ} 32^{\prime} \mathrm{W}$ & Southeastern Bering Sea & 1 & K. Williams, unpub. & Sighting K. Williams, NMFS Platform of Opportunity data, photographed \\
\hline
\end{tabular}

Table 2.3

Sightings of right whales in the North Pacific, with location not specified.

\begin{tabular}{|c|c|c|c|c|c|c|}
\hline Date & Latitude & Longitude & Location & No. & Source & Remarks \\
\hline 1954 to ? & - & - & North Pacific, north of $40^{\circ} \mathrm{N}$ & 20 & Ivashin and Rovnin, 1967 & All marked; no details given \\
\hline 1958-1962 & - & - & 'Pelagic' region & 193 & Omura et al., 1969, table 14 & $\begin{array}{l}\text { Japanese catcherboat sightings; table } 14 \text { shows } 310 \text { sightings, but } 117 \text { for which details are available from } \\
\text { unpublished ICR data sources are given separately above }\end{array}$ \\
\hline 1963 & - & - & 'Northern North Pacific' & 49 & Omura et al., 1969, table 15 & $\begin{array}{l}\text { May include } 3 \text { whales killed as scientific research take. Table } 15 \text { includes whales in other years, presumably } \\
\text { duplicating those listed in table } 14 \text { and noted above }\end{array}$ \\
\hline 1966-1967 & - & - & 'Pelagic' region & 22 & Omura et al., 1969 & Japanese catcherboat sightings \\
\hline
\end{tabular}

Notes: Two reports of 8 right whales observed off Washington state in 1959 by Fiscus and Niggol (1965) have been excluded because of the unreliable nature of the sightings; see the critique of Scarff (1986a, p.52). Nasu (1960) reports 2 right whales in the Chukchi and northern Bering seas in Aug 1958, but these were probably bowheads.

Berzin and Doroshenko (1982) note a 'recent' sighting at 58³0'N (longitude given in Berzin and Rovnin 1966 as 167³2'W), with no further details.

Possible but unconfirmed sightings of right whales are reported in Zenkovich (1934), Klumov (1962), DeBus (1975), Morriset al. (1983, p.141), Reeves and Leatherwood (1985), Scarff (1986a), and Blokhin (1988).

Several other tentative sightings were recorded between 1959 and 1989 by NMFS Platform of Opportunity data. 
Table 2.4

Summary of sightings since 1900 by area. Excluded are 284 sightings with no location specified. The $41 \mathrm{NE}$ Pacific sightings include 16 reported only as 'East of $180^{\circ}$ '

\begin{tabular}{lrlr}
\hline Western North Pacific & \multicolumn{3}{l}{ Eastern North Pacific } \\
\hline Japan & 370 & Northwest Ground & $177^{1}$ \\
Kuril Islands & 331 & West Coast & 24 \\
Okhotsk Sea & 195 & Bering Sea & 178 \\
NW Pacific & 69 & NE Pacific & 41 \\
Western Aleutians & 23 & Eastern Aleutians & 269 \\
& & Hawaii & 4 \\
Total & 988 & Total & 693 \\
\hline
\end{tabular}

${ }^{1}$ This total includes sightings in 1958-64 reported by Berzin and Rovnin (1966) but not the 200 animals reported for 1963 by Berzin and Doroshenko (1982). See text for discussion of the conflict between these two papers.

The latter report represents a striking anomaly in an area where subsequent sightings of this species have been rare, and have generally been of single animals. Data given in Wada (1975) for Japanese sighting cruises made between 1965 and 1973 revealed no right whale sightings in the area of the Soviet sighting, and a total of only ten whales in the survey blocks that surround it for several hundred miles in all directions. More significantly, Berzin and Rovnin's (1966) plots of sightings from the same source show a scatter of animals across the northeastern Pacific, with no concentrations near the position given by Berzin and Doroshenko (1982). Finally, although the English translation of Berzin and Rovnin (1966) could be verified, Berzin and Doroshenko (1982) was submitted only in English and we could not locate the original Russian manuscript to check various details. In light of all this, and the seeming improbability of the existence of such a large concentration of right whales, it has been assumed that the scattered distribution shown by Berzin and Rovnin (1966) is accurate. There appears no way of determining the extent to which these data include duplicate sightings or other errors, and they are reported with this caveat.

\section{Catches}

In all, 741 right whales are recorded as being caught for either scientific or commercial purposes since 1900. Of these, 330 were killed in the western North Pacific (Table 3.1), 160 of which were taken in the waters of Japan and the Okhotsk Sea by the Japanese (Omura, 1986). A total of 411 were killed in the eastern North Pacific (Table 3.2). Twenty-eight were taken in the Gulf of Alaska or eastern Bering Sea between 1911 and 1938. Eleven were taken by Japanese and Soviet whalers as scientific catches, but the remaining 372 were killed during Soviet illegal pelagic whaling from the Bering Sea and Gulf of Alaska. There is only a single catch record from the west coast of the continental United States: a whale killed on 9 April 1924 near the Farallon Islands off central California (Gilmore, 1956). Another whale was accidentally killed off the northwest coast of Vancouver Island, Canada in May 1951 (Pike and MacAskie, 1969). The single right whale killed on 1 June 1964 (Table 3.1) by the USSR and reported as an infraction is included in the 1964 illegal USSR catches from the Gulf of Alaska.

A number of sources list animals taken off the northwestern coast of North America and give different numbers. Kellogg (1931) reports 17 right whales taken; one of which was reported killed off British Columbia in 1924 but is not included in the total of 27 given for the period
1914-1935 by Reeves et al. (1985). We have not found this whale in original record summaries for British Columbia and believe Kellogg to be in error. Tomilin (1957) reported that 28 right whales were killed in this region between 1911 and 1938; however, he provided neither details nor sources. Our records for this same period agree with Tomilin.

Other right whales may have been illegally caught but not recorded by the Soviet Union in the western North Pacific. For example, Yablokov (1994) noted that right whales had been taken in the late 1950 s by a whaling station on Paramushir Island in the northern Kurils.

Among the recorded catches, 23 were animals taken for the purpose of scientific research (9 and 14 in the eastern and western North Pacific, respectively). All of these have been previously reported, including ten taken by the USSR (Klumov, 1962), and 13 by Japan (Omura, 1958; Omura et al., 1969).

Best (1987) estimated that seven right whales were taken by American whalers throughout the North Pacific between 1900 and 1909. However, this figure does not come from specific catch records; rather, it was calculated based upon imports of oil and baleen during this period. Consequently, they have not been incorporated into the present review.

\section{Strandings and entanglements}

The 13 records of strandings and entanglements are listed in Table 4; all but one are from the western North Pacific. Five of the 12 western records are from the Commander Islands, three from Kamchatka, two from Japan, and one each from the Kuril Islands and Sakhalin Island. The sole eastern North Pacific record, from California, dates from 1916.

\section{DISCUSSION}

\section{Population structure and migration \\ Distribution}

Nineteenth-century whaling records show that right whales were once abundant across much of the North Pacific (Maury, 1852; 1853; Townsend, 1935; Scarff, 1986a; 1991). Areas of concentration included Japan, the Okhotsk Sea, the Kurils, Kamchatka, the Aleutians and southeastern Bering Sea (Bristol Bay Ground), and the Northwest Ground.

The data summarised here generally confirm this distribution. In the western North Pacific, right whales have been observed in significant numbers within the last forty years in areas known to be historically important. In particular, the Okhotsk Sea has consistently had significant numbers of sightings, the most during the 1990s; it is clear that this region, and the adjacent Kuril Islands and Kamchatka coast, represent a major feeding ground for the species. Furthermore, a concentration of Japanese sightings in the Bering Sea, loosely centered around $55^{\circ} \mathrm{N}, 170^{\circ} \mathrm{W}$, suggests that this region was an important summer habitat for eastern North Pacific right whales. Small numbers of right whales have been sighted east of this area in recent years (1998-1999) during dedicated vessel and aerial surveys (LeDuc et al., 2000). A detailed Geographic Information Systems analysis of all data presented here is in preparation.

The current rarity of right whales in previously populous parts of the eastern North Pacific is testament to the extreme damage done by whaling. Nowhere is the contrast between past and present abundance more striking than on the Northwest Ground. Scammon (1874) noted that right whales were there "scattered... as far as the eye can discern from the 
Table 3.1

Commercial and scientific catches of right whales in the western North Pacific since 1900.

\begin{tabular}{|c|c|c|c|c|c|c|c|}
\hline Date & Latitude & Longitude & Location & No. & Source & Nation & Remarks \\
\hline \multirow[t]{7}{*}{ 1911-1948 } & & & Kuril Is, S Hokkaido and & 113 & Omura, 1986 & Japan & \\
\hline & & & NE Honshu & 26 & & & \\
\hline & & & S Honshu and E Kyushu & 8 & & & \\
\hline & & & Bonin Is & 8 & & & \\
\hline & & & Okhotsk Sea & 4 & & & \\
\hline & & & W Kyushu & 1 & & & \\
\hline & & & Coast of Korea & & & & \\
\hline Feb. 1921 & $28^{\circ} 00^{\prime} \mathrm{N}$ & $129^{\circ} 24^{\prime} \mathrm{E}$ & Amami Is, Japan & 1 & $\begin{array}{l}\text { Miyazaki and } \\
\text { Nakayama, } 1989\end{array}$ & Japan & $47 \mathrm{ft}$ - stranding? \\
\hline $1924-1925$ & & & Off Kamchatka & 2 & Tomilin, 1957 & Norway & Factory ship Kommandøren I \\
\hline $1932-1946$ & & & WN Pacific and Bering Sea & 9 & Zenkovich, 1955 & USSR & Factory ship Aleut \\
\hline Aug. 1940 & & & Bering Sea & 1 & Terry, 1950 & Japan & Factory ship Tonan maru \\
\hline 10-11 Jun. 1941 & $48^{\circ} \mathrm{N}$ & $158-159^{\circ} \mathrm{E}$ & SE of Kamchatka & 2 & $\begin{array}{l}\text { Matsuura and Maeda, } \\
\text { 1942; Omura, } 1958\end{array}$ & Japan & $\begin{array}{l}\text { Factory ship Tonan maru. } 58 \mathrm{ft} \text { female, } \\
45 \mathrm{ft} \text { male }\end{array}$ \\
\hline 1941 & & & Off Kamchatka & 1 & Terry, 1950 & Japan & Factory ship Tonan maru \\
\hline 17 May 1955 & $45^{\circ} 08^{\prime} \mathrm{N}$ & $149^{\circ} 46^{\prime} \mathrm{E}$ & Kuril Is & 1 & Klumov, 1962 & USSR & Scientific research take; $18.3 \mathrm{~m}$ female \\
\hline 1 Jun. 1955 & $46^{\circ} 23^{\prime} \mathrm{N}$ & $152^{\circ} 34^{\prime} \mathrm{E}$ & Kuril Is & 1 & Klumov, 1962 & USSR & Scientific research take; $17 \mathrm{~m}$ male \\
\hline 19 Jun. 1955 & $47^{\circ} 01^{\prime} \mathrm{N}$ & $150^{\circ} 25^{\prime} \mathrm{E}$ & Kuril Is & 1 & Klumov, 1962 & USSR & Scientific research take; $16.3 \mathrm{~m}$ female \\
\hline 13 Jul. 1955 & $49^{\circ} 44^{\prime} \mathrm{N}$ & $157^{\circ} 17^{\prime} \mathrm{E}$ & Kuril Is & 1 & Klumov, 1962 & USSR & Scientific research take; $17.1 \mathrm{~m}$ male \\
\hline 22 Jul. 1955 & $49^{\circ} 34^{\prime} \mathrm{N}$ & $156^{\circ} 35^{\prime} \mathrm{E}$ & Kuril Is & 1 & Klumov, 1962 & USSR & Scientific research take; $17.4 \mathrm{~m}$ female \\
\hline 22 Jul. 1955 & $49^{\circ} 42^{\prime} \mathrm{N}$ & $154^{\circ} 31^{\prime} \mathrm{E}$ & Kuril Is & 1 & Klumov, 1962 & USSR & Scientific research take; $19 \mathrm{~m}$ male \\
\hline 10 Aug. 1955 & $50^{\circ} 47^{\prime} \mathrm{N}$ & $155^{\circ} 21^{\prime} \mathrm{E}$ & Kuril Is & 1 & Klumov, 1962 & USSR & Scientific research take; $16.6 \mathrm{~m}$ male \\
\hline 10 Aug. 1955 & $50^{\circ} 22^{\prime} \mathrm{N}$ & $155^{\circ} 12^{\prime} \mathrm{E}$ & Kuril Is & 1 & Klumov, 1962 & USSR & Scientific research take; $16.6 \mathrm{~m}$ male \\
\hline 11 Aug. 1955 & $51^{\circ} 05^{\prime} \mathrm{N}$ & $155^{\circ} 51^{\prime} \mathrm{E}$ & Kuril Is & 1 & Klumov, 1962 & USSR & Scientific research take; $11.4 \mathrm{~m}$ female \\
\hline 28 Aug. 1955 & $50^{\circ} 00^{\prime} \mathrm{N}$ & $154^{\circ} 25^{\prime} \mathrm{E}$ & Kuril Is & 1 & Klumov, 1962 & USSR & Scientific research take; $17.8 \mathrm{~m}$ female \\
\hline May-Jun. 1956 & $38-42^{\circ} \mathrm{N}$ & $143-149^{\circ} \mathrm{E}$ & Off eastern Japan & 2 & Omura, 1957 & Japan & $\begin{array}{l}\text { Scientific research take; } 12.4 \mathrm{~m} \text { male, } \\
12.6 \mathrm{~m} \text { female }\end{array}$ \\
\hline Late 1950’s & & & Off Paramushir I., Kuril Is & $?$ & Yablokov, 1994 & USSR & $\begin{array}{l}\text { Number unknown, but Yablokov had } \\
\text { 'anatomical materials' from right } \\
\text { whales taken at this time. }\end{array}$ \\
\hline 1967 & & & $\begin{array}{l}\text { Off SE Sakhalin I., Okhotsk } \\
\text { Sea }\end{array}$ & 126 & Doroshenko, 2000 & USSR & Illegal pelagic whaling \\
\hline 1967 & & & $\begin{array}{l}\text { Olyutorskiy Bay, } \\
\text { Kamchatka }\end{array}$ & 1 & Doroshenko, 2000 & USSR & \\
\hline 20/25 Jul. 1968 & $48^{\circ} \mathrm{N}$ & $145-146^{\circ} \mathrm{E}$ & $\begin{array}{l}\text { Southeastern Sakhalin I., } \\
\text { Okhotsk Sea }\end{array}$ & 2 & Omura et al., 1969 & Japan & $\begin{array}{l}\text { Scientific research take; } 15.2 \mathrm{~m} \text { male, } \\
12.6 \mathrm{~m} \text { female }\end{array}$ \\
\hline 1971 & & & Northern Kuril Is & 10 & Doroshenko, 2000 & USSR & Illegal pelagic whaling \\
\hline Jan. 1973 & & & Haiyang I., Yellow Sea & 2 & Wang, 1978 & China & $\begin{array}{l}18 \mathrm{~m} \text { female, } 12.8 \mathrm{~m} \text { male. In Dailan } \\
\text { Museum of Natural History }\end{array}$ \\
\hline Mid-Oct. 1974 & & & Sea of Japan & 1 & Park, 1987 & Korea & $\begin{array}{l}\text { 'Approx } 64 \mathrm{ft} \text { '. Taken by whaling ship } \\
\text { Je } 3 \text { Deyahng }\end{array}$ \\
\hline Dec. 1977 & & & SE Haiyang I., Yellow Sea & 1 & Wang, 1988 & China & $17.1 \mathrm{~m}$ female with $4.99 \mathrm{~m}$ foetus \\
\hline
\end{tabular}


Table 3.2

Commercial and scientific catches of right whales in the eastern North Pacific since 1900.

\begin{tabular}{|c|c|c|c|c|c|c|c|}
\hline Date & Latitude & Longitude & Location & No. & Source & Nation & Remarks \\
\hline \multirow[t]{4}{*}{$1911-1938$} & & & Gulf of Alaska, SE. Bering Sea & 28 & Tomilin, 1957 & USA & Years and total of 28 taken from \\
\hline & & & and off British Columbia & & Reeves et al., 1985 & Canada & Tomilin; others give fewer \\
\hline & & & & & Brueggeman et al., 1986 & & animals over shorter period. \\
\hline & & & & & Kellogg, 1931 & & See text. \\
\hline \multirow[t]{2}{*}{9 Apr. 1924} & $37^{\circ} 40^{\prime} \mathrm{N}$ & $124^{\circ} \mathrm{W}$ & Off Farallon Is, California & 1 & Gilmore, 1956 & USA & Moss Landing whaling station \\
\hline & & & & & & & $\begin{array}{l}\text { log says } 40 \mathrm{ft} \text { female, empty } \\
\text { stomach. }\end{array}$ \\
\hline \multirow{2}{*}{$\begin{array}{r}\text { May } 1951 \\
1961\end{array}$} & & & Off British Columbia & 1 & Pike and McAskie, 1969 & Canada & 'accident' \\
\hline & $55^{\circ} 54^{\prime} \mathrm{N}$ & $153^{\circ} 07^{\prime} \mathrm{W}$ & South of Kodiak I., Alaska & 1 & Omura et al., 1969 & Japan & Scientific research take \\
\hline 1961 & $55^{\circ} 54^{\prime} \mathrm{N}$ & $153^{\circ} 08^{\prime} \mathrm{W}$ & South of Kodiak I., Alaska & 1 & Omura et al., 1969 & Japan & \\
\hline 1961 & $55^{\circ} 53^{\prime} \mathrm{N}$ & $153^{\circ} 06^{\prime} \mathrm{W}$ & South of Kodiak I., Alaska & 1 & Omura et al., 1969 & Japan & \\
\hline 1962 & $53^{\circ} 42^{\prime} \mathrm{N}$ & $171^{\circ} 17^{\prime} \mathrm{W}$ & SE Bering Sea (north of Aleutians) & 1 & Omura et al., 1969 & Japan & $\begin{array}{l}\text { Scientific research take; TK on } \\
\text { factory ship }\end{array}$ \\
\hline 1962 & $54^{\circ} 30^{\prime} \mathrm{N}$ & $170^{\circ} 22^{\prime} \mathrm{W}$ & SE Bering Sea (north of Aleutians) & 1 & Omura et al., 1969 & Japan & \\
\hline 1962 & $54^{\circ} 18^{\prime} \mathrm{N}$ & $170^{\circ} 21^{\prime} \mathrm{W}$ & SE Bering Sea (north of Aleutians) & 1 & Omura et al., 1969 & Japan & \\
\hline 1963 & $53^{\circ} 52^{\prime} \mathrm{N}$ & $172^{\circ} 46^{\prime} \mathrm{W}$ & SE Bering Sea (north of Aleutians) & 1 & Omura et al., 1969 & Japan & Scientific research take \\
\hline 1963 & $54^{\circ} 04^{\prime} \mathrm{N}$ & $172^{\circ} 35^{\prime} \mathrm{W}$ & SE Bering Sea (north of Aleutians) & & Omura et al., 1969 & Japan & \\
\hline 1963 & $54^{\circ} 03^{\prime} \mathrm{W}$ & $172^{\circ} 50^{\prime} \mathrm{W}$ & SE Bering Sea (north of Aleutians) & & Omura et al., 1969 & Japan & \\
\hline 1963 & & & Gulf of Alaska & 141 & Doroshenko, 2000 & USSR & Illegal pelagic whaling \\
\hline 1 Jun. 1964 & $57^{\circ} 20^{\prime} \mathrm{N}$ & $150^{\circ} 00^{\prime} \mathrm{W}$ & Gulf of Alaska, E of Kodiak I. & $1^{*}$ & IWC database & USSR & $\begin{array}{l}\text { Infraction. Factory ship Dalnij } \\
\text { Vostok. } 13.8 \mathrm{~m} \text { male }\end{array}$ \\
\hline 1964 & & & Gulf of Alaska & 87 & Doroshenko, 2000 & USSR & Illegal pelagic whaling \\
\hline 1964 & & & SE Bering Sea & 113 & Doroshenko, 2000 & USSR & Illegal pelagic whaling \\
\hline 1965 & & & Gulf of Alaska & 20 & Doroshenko, 2000 & USSR & Illegal pelagic whaling \\
\hline 1966 & & & Gulf of Alaska & 3 & Doroshenko, 2000 & USSR & Illegal pelagic whaling \\
\hline 1967 & & & SE Bering Sea & 8 & Doroshenko, 2000 & USSR & Illegal pelagic whaling \\
\hline
\end{tabular}

* This whale is not included in the total count as it is considered part of the 87 whales illegally taken in the Gulf of Alaska in 1964 .

masthead'. Remarkably few right whales have been observed in these waters in the past thirty-five years despite often extensive effort in the form of dedicated marine mammal surveys.

It has been suggested by several researchers that the current paucity of sightings from waters off the west coast of North America is due to overexploitation (e.g. Gaskin, 1987). However, Scarff (1986a; 1991) makes a convincing case that right whales were never common in this region. Although some records are available from Washington state, there is little evidence that right whales were regularly taken by local aboriginal peoples. Relatively few right whales were observed or killed by whalers on this coast in even the early years of the fishery (Townsend, 1935; Scarff, 1986a; Mitchell and Reeves, 2001). Furthermore, as Scarff (pers. comm.) points out, there is not a single record of a calf, either at sea or stranded, from this region (this is not the case for the western North Pacific).

\section{Migratory movement, breeding and calving}

The seasonal migratory movement of right whales in the North Pacific has been a topic of debate for many years. In general, one observes a northward movement to high latitudes in spring, and a similar southward trend in autumn (although there is considerably less information for the latter period). However, right whales are found across a broad latitudinal range during both seasons, suggesting a staggered migration (Scarff, 1991).

A more fundamental question relates to the location of breeding and calving grounds. In the western North Pacific, various areas have been proposed, including the Ryukyu Islands (Omura, 1986), the Yellow Sea (Tomilin, 1957), the Sea of Japan (Omura, 1986), and offshore waters far from land (Scarff, 1991). There are no recent reports of right whales around Taiwan, although Townsend (1935) plotted a small number of catches in the Taiwan Strait. The Bonin Islands have also been proposed as a wintering area (Omura, 1958); the three recent sighting records from this area, all made in April, are of interest in this regard, although Scarff (1991) notes that Maury recorded few right whales in this area despite reasonable search effort in late winter. Overall, mid-winter sightings and seasonal movements in spring and autumn give various degrees of support to all of the above suggestions, but the general paucity of records from winter make a definitive assessment impossible.

There is very little information on where right whales from the eastern North Pacific spend their winters. The 14 sightings (of a total of 15 animals) from California and Baja California this century exhibit seasonality: with the exception of one September record, all occurred between February and May. While this could be viewed as a remnant population continuing to follow traditional routes to or from an unknown wintering area off the west coast, the historical data do not support the idea that this region ever contained major habitats for right whales at any time of year. Based upon some autumn and spring records in mid-ocean in Maury's compilations, Scarff (1991) argues for more rigorous examination of the possibility that the animals which summered along the high-latitude margins on the eastern North Pacific wintered and calved in mid-ocean waters far offshore. Others have suggested that right whales on both sides of the North Pacific calve (or once calved) in embayments, as occurs in the North Atlantic and in southern right whales (Eubalaena australis). However, no such bay has been identified. Furthermore, in areas where coastal calving or nursing takes place (e.g. western North Atlantic, Kraus et al., 1986; Argentina, Payne, 1986), not all females appear to use these habitats, and some may calve offshore. Since the advantage of coastal calving is not clear, there is currently no good reason to suppose that right whales would not give birth and perhaps mate far from land. 


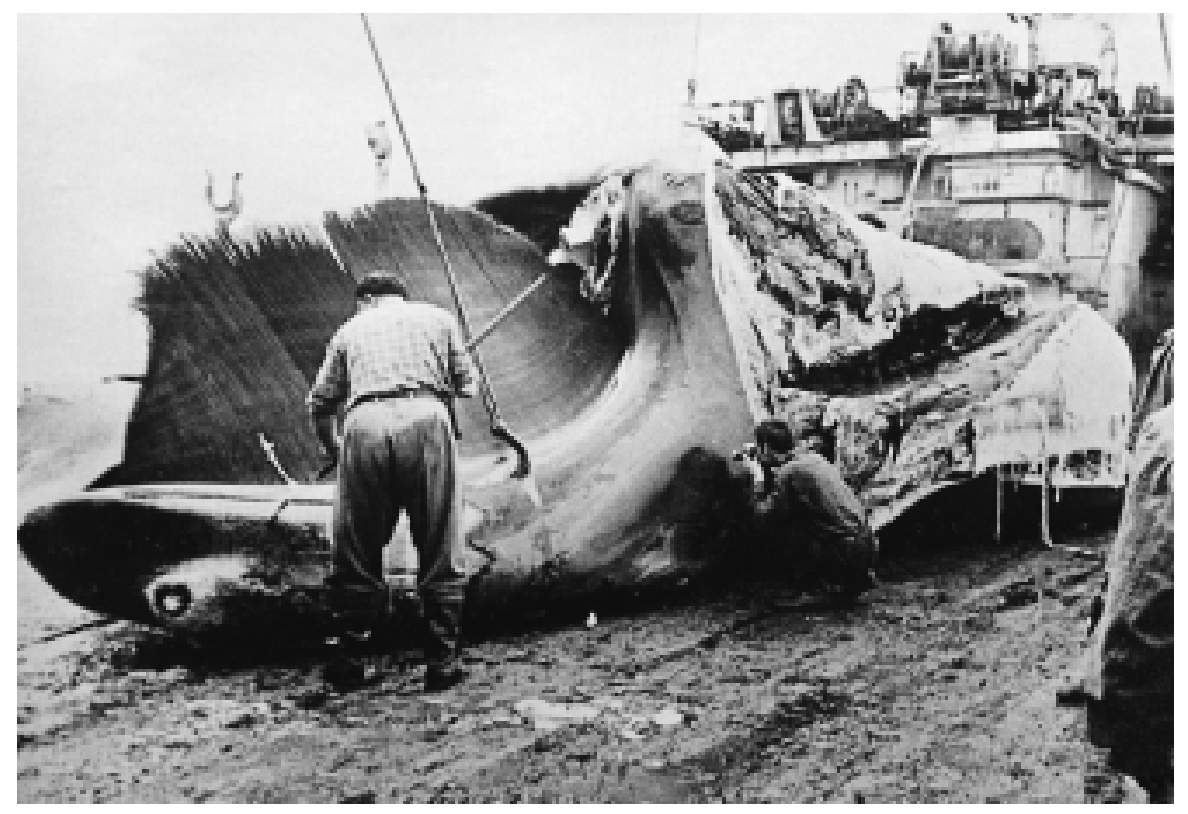

Fig. 1. North Pacific right whale taken in the southeastern Bering Sea (Bristol Bay Grounds) in 1964 and being processed on board the USSR factory ship the Vladivostok. Courtesy of A.A. Berzin.
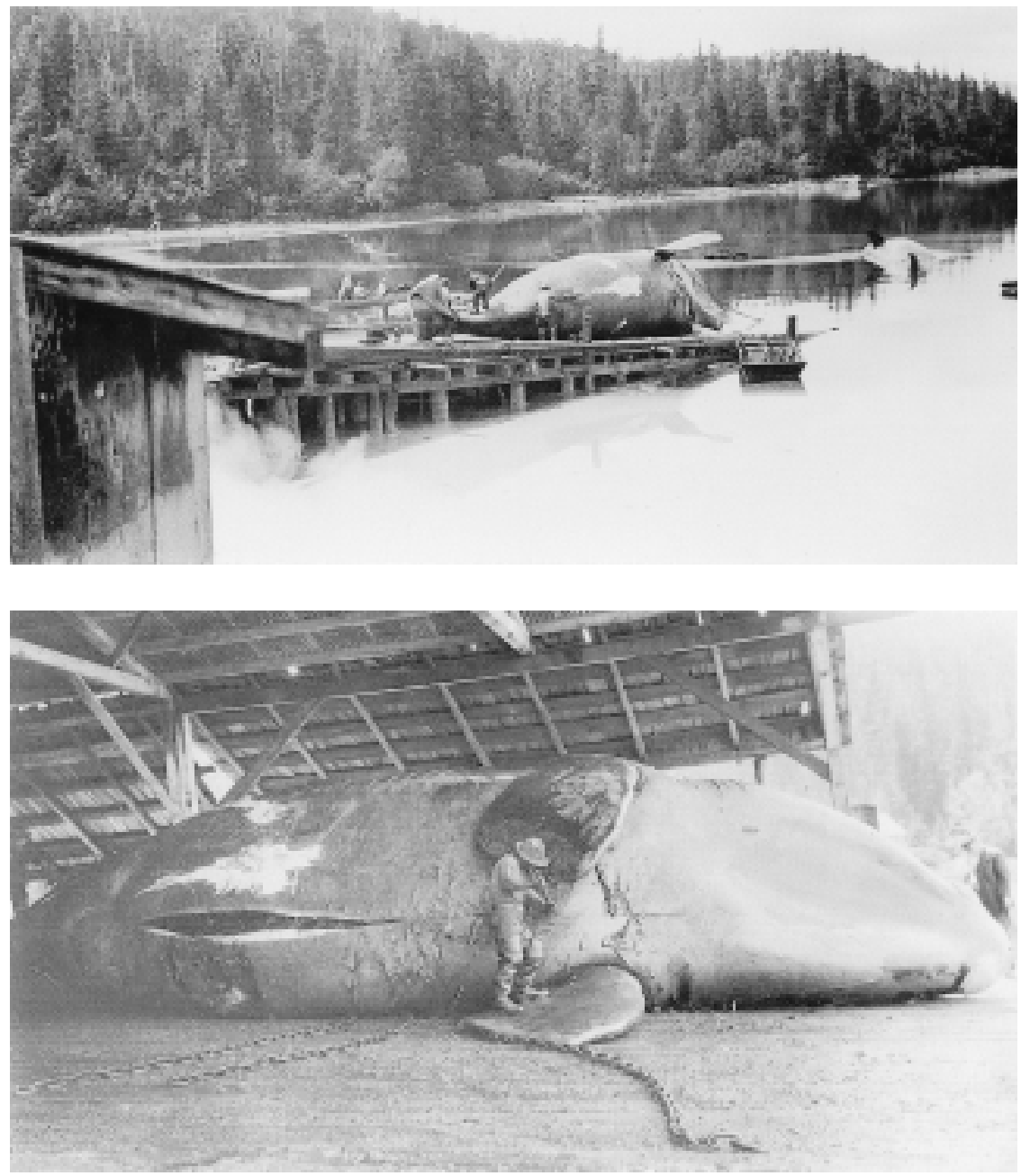

Fig. 2. North Pacific right whale landed on 25 June 1929 at Rose Harbour, northern end of Kunghit Island, Queen Charlotte Islands, Canada. Courtesy of J. Storrie via J. Goddard and the Maritime Museum of British Columbia, Victoria, BC, Canada. 


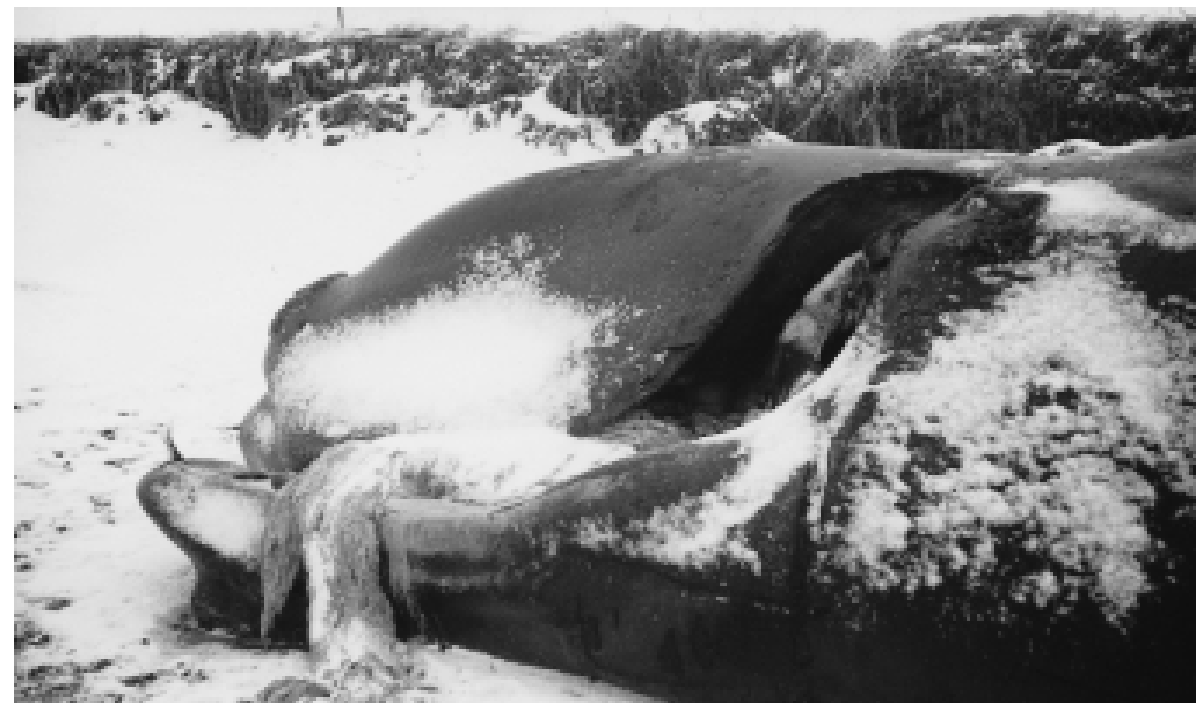

Fig. 3. North Pacific right whale found stranded 14 November 1999 on the west coast of Kamchatka, Russia. Courtesy of V.S. Nikulin.

Table 4

Strandings and entanglements of North Pacific right whales since 1900 .

\begin{tabular}{|c|c|c|c|c|c|}
\hline Date & Latitude & Longitude & Location & Source & Remarks \\
\hline 1902 & & & Hamiyashima, Amami I., Japan & Miyazaki and Nakayama, 1989 & Stranded \\
\hline 14 Nov. 1916 & & & Santa Cruz I., California & Woodhouse and Strickley, 1982 & Stranded \\
\hline Dec. 1939 & & & Lundskii Bay, Sakhalin I. & Tomilin, 1957 & Stranded $c a 9 \mathrm{~m}$ \\
\hline 1976 & & & Bering I. (Commander Is) & Ivashin and Vertjankin, 1987 & Stranded female \\
\hline 15 Apr. 1977 & $34^{\circ} 40^{\prime} \mathrm{N}$ & $138^{\circ} 45^{\prime} \mathrm{E}$ & Kumomi, Izu Peninsula Japan & Yamamoto and Hiruta, 1978 & Stranded $11.5 \mathrm{~m}$ male \\
\hline 1980 & & & Bering I. (Commander Is) & A.M. Burdin, pers. comm. & Stranded? \\
\hline 15 Mar. 1984 & & & Bering I. (Commander Is) & A.M. Burdin, pers. comm. & Stranded male \\
\hline 25 Jul. 1984 & & & Medny I. (Commander Is) & A.M. Burdin, pers. comm. & Stranded? \\
\hline 16 Oct. 1989 & & & Pacific coast of C Lopatka, Kamchatka & Kornev, 1994 & $\begin{array}{l}\text { Stranded } 12.15 \mathrm{~m} \text { male, entangled } \\
\text { in gillnet }\end{array}$ \\
\hline 25 Jun. 1991 & & & Medny I. (Commander Is) & A.M. Burdin, pers. comm. & Stranded, size and sex unknown \\
\hline 29 Mar. 1997 & & & Cape Lopatka, Kamchatka & V.S. Nikulin, pers.comm. & Stranded, $c a 14 \mathrm{~m}$ \\
\hline Summer 1998 & & & Shumshu I. , Kuril Is & S.I. Kornev, pers. comm. & Stranded, size and sex unknown \\
\hline 14 Nov. 1999 & & & West coast of Kamchatka & V.S. Nikulin, pers. comm. & Stranded, $12.6 \mathrm{~m}$ male \\
\hline
\end{tabular}

Note: On 27 Jan 1995 a right whale skull was unearthed at a beach development 2 miles north of Crescent City, California (41 $\left.{ }^{\circ} 46^{\prime} \mathrm{N}, 124^{\circ} 15^{\prime} \mathrm{W}\right)$. Its date of burial and the animal's cause of death were unknown (J. Cordaro, pers. comm.)

\section{Stock separation}

The question of whether two or more stocks of right whales exist in the North Pacific remains open. Townsend's (1935) charts show a largely discontinuous distribution of right whales across high latitudes, with few catches in the mid-Pacific region. This has been used by a number of authors as evidence of the existence of two discrete populations (e.g. Klumov, 1962). However, Scarff (1991) noted that Maury's charts showed a relatively continuous distribution across the Pacific, including a surprisingly high (relative to effort) incidence of sightings in mid-ocean. Scarff maintained that the gaps in Townsend's charts result from a lack of searching effort in the regions concerned, and that the relatively low number of catches from the central Aleutians region was due to the whalers' concentration on known high-density areas elsewhere.

Twentieth century sightings support the two-stock hypothesis. The fact that the right whale populations in the eastern and western North Pacific appear to have distinct catch and recovery histories also supports the idea that at least two stocks exist, at least with regard to feeding ground divisions. The eastern population was clearly the more intensively whaled, and this is reflected in the fact that fewer animals exist there today than in the west. This difference in post-exploitation abundance represents some evidence in favour of the two-stock hypothesis. However, the extent of exchange between the two populations in high latitudes, and whether the different feeding stocks mix on a common breeding ground offshore, is unknown. This could be addressed with molecular genetic analyses using both mitochondrial and microsatellite DNA, although finding and sampling a sufficient number of whales (notably in the east) would be a major obstacle to such a study.

A second question relates to possible subdivision within the western North Pacific. Both Klumov (1962) and Omura (1986) believed that the right whales which summer in the Okhotsk Sea represent a discrete population which winters in the Sea of Japan and perhaps the East China Sea. However, right whales today in the Sea of Japan appear to have been almost extirpated. Omura (1986) believed that a second, 'Pacific', stock migrates up the east coast of Japan, possibly from breeding grounds in the Ryukyu Islands, and summers in the Kurils and the Bering Sea. Although we find it difficult to accept that animals found in such close proximity to one another (notably in the Kurils and Okhotsk Sea) could represent discrete stocks, we can offer no new data to this debate.

\section{Extent of illegal catches}

In order to address the question of the present status of North Pacific right whales, the extent of illegal hunting by the Soviet Union must be examined. Three known episodes can 
be identified: factory ship whaling in the Okhotsk Sea and the Northwest Ground/southeastern Bering Sea, and a land-based operation in the Kurils. Based upon the evidence presented below, it seems probable that many of the Soviet 'sightings' of right whales in these areas are in fact whales that were killed.

\section{Southeastern Bering Sea/Northwest Ground/eastern North Pacific}

As noted above, there is considerable confusion concerning exactly where the Soviets observed 200 right whales in 1963, and whether this uncharacteristically large number can be considered accurate. However, the more significant issue concerns whether these sightings (wherever they were made), and others reported in 1958-64 by Berzin and Rovnin (1966), actually represent catches. It should be noted that the Soviet catches in the southeastern Bering Sea and in the Gulf of Alaska in 1964 totalled 200 right whales (Doroshenko, 2000). A.A. Berzin (pers. comm. to RLB) reported that two sister ships built specifically for the North Pacific (the Vladivostok and the Dalniy Vostok) killed about 200 right whales in the eastern North Pacific in 1964.

The Soviet catch of 372 right whales during the 1960s must have represented a large proportion, probably the majority, of the remaining eastern North Pacific population. If so, one would expect to see this depletion reflected in data from subsequent years. This appears to be the case. In addition to the Soviet sightings of several hundred animals in 1958-64 (Berzin and Rovnin, 1966), analysis of Japanese whalecatcher observations from 1954 to 1957 show more than a hundred sightings of right whales in the eastern Aleutians and southeastern Bering Sea in the months of June and July, including approximately 60 in July of 1956 alone (Omura, 1958, fig. 1). Overall, between 1941 and 1964 there were 598 sightings of right whales east of $180^{\circ}$ (including a midpoint total of 330 for Berzin and Rovnin, 1966, and ignoring the 200 from Berzin and Doroshenko, 1982); this is an average of 24.9 whales per year. These records contrast sharply with the results of surveys conducted in later years: from 1965 to 1999 , only 82 sightings were reported for the entire eastern North Pacific, or 2.3 per year. This number includes the results of extensive Japanese surveys conducted over a 27-year period from 1965 to 1991 , including in the previously quite populous eastern Aleutians and southeastern Bering Sea. Even if all of the sightings reported by Berzin and Rovnin (1966) were false, a major decline is still evident.

As early as the mid-1970s, Gilmore (1978) suggested that illegal catches were made on the Northwest Ground. However, information to support his suspicion was not available until very recently and emerged in part because of the present review. In conclusion, it is clear that the Soviet takes inflicted severe damage on the remaining eastern North Pacific population.

\section{Kuril Islands/Okhotsk Sea}

Yablokov (1994) stated that 'hundreds' of right whales (including bowheads) were taken in the Okhotsk Sea in the 1960s, and also mentions whaling of undetermined extent from Paramushir Island in the Kurils prior to the late 1950s (this whaling was in addition to the scientific research take of ten animals reported by Klumov, 1962). Since Paramushir was just one of several whaling stations that began operations in the Kurils in 1948, it is possible that the 1950s takes were also extensive.
Available data show that the take of right whales in this region was indeed substantial. As in the eastern North Pacific, the sighting data for this area reveal a pattern of relative abundance followed by apparent decline. In just three summers (1955-57), 244 right whale sightings were reported by Soviet whaling vessels in the Kurils alone (Klumov, 1962), although the actual number of whales or of the rate of duplicate sightings involved is unknown. In the years that followed, there were only two substantial reports of right whales in the Okhotsk Sea region: one of 70 animals off eastern Sakhalin in 1967 (Berzin and Vladimirov, 1989) and another of 40-45 northeast of Kashevarov Bank in 1974. In 1967, 126 right whales were killed by Soviet commercial whaling operations off the southeastern end of Sakhalin Island (Doroshenko, 2000). In July 1968, the Japanese killed two right whales in the same area under a research whaling permit (Omura et al., 1969). By contrast, between 1975 and 1991 , only nine animals were recorded in the region by either Soviet or Japanese surveys, although 34 were observed in the summer of 1992 and 11 in the summer of 1999. Although the trend is less marked than in the eastern North Pacific, the general decline in sightings after the 1960s suggests that a significant proportion of the right whales of the Okhotsk Sea region were killed during one or more periods of illegal whaling by the USSR. How many of the various Soviet sightings (including the 244 reported from the 1950s by Klumov) may represent catches is unclear, and no original data appear to exist from this period.

\section{Present status}

Regrettably, none of the published estimates of abundance relating to North Pacific right whales can be regarded as reliable. Even the indices of abundance calculated from extensive Japanese sighting surveys (e.g. Ohsumi and Wada, 1974) suffer from the inevitable problems of high variance that accompany extrapolations from very few observations over a wide area. Other estimates appear to be little more than conjecture based upon general patterns of sightings: examples include '300-500' for the North Pacific (Berzin and Yablokov, 1978), 100-200 for the North Pacific (Braham and Rice, 1984) and 150-200 for the Okhotsk Sea (Berzin and Vladimirov, 1989, citing Berzin, 1982), or 800 and 900 for the Okhotsk Sea (Vladimirov, 1994 and Vladimirov, 2000, respectively). However, no quantitative data exist to confirm any of these estimates. The most refined preliminary estimate for the Okhotsk Sea is 900 whales (Miyashita and Kato, 1998), but the confidence interval for this estimate $(404-2,108)$ is large. The only thing common to all of the estimates, whether regional or basin-wide, is that they are low: all agree that the North Pacific right whale is not numerous anywhere within its historic range.

The data summarised here clearly support this view. The relative paucity of sightings virtually everywhere in the $20^{\text {th }}$ century, and the pattern of apparent decline observed after the 1960s, all point to a situation in which remnant populations may have been slowly recovering from intensive whaling, only to be devastated by illegal Soviet catches. In the western North Pacific, Soviet catches nullified any increases that occurred during the $20^{\text {th }}$ century. Although quantitative analysis is impossible, recent sightings suggest that this population may number at least in the low hundreds and may therefore be large enough to survive. By contrast, sightings of right whales in the eastern North Pacific are today exceedingly rare; this is true despite the often intensive search effort that has occurred in many potential right whale habitats as a result of offshore oil and gas development, and recent dedicated surveys. Overall, the situation in the North 
Pacific closely parallels that with the North Atlantic right whale. The eastern North Atlantic stock was greatly reduced by protracted whaling; it appears likely that the remaining animals were then virtually extirpated by a burst of Norwegian catches at the turn of the $20^{\text {th }}$ century (Collet, 1909; Brown, 1986). A remnant population numbering approximately 300 animals remains in the western North Atlantic (IWC, 2001a).

It is not clear what the future holds for the right whale in the North Pacific. It is possible that the western population is large enough that, given sufficient time and protection, it will recover. However, one should note that no increase has been apparent in a population of similar size in the western North Atlantic despite six decades of protection, although this population is known to suffer a high mortality rate from anthropogenic factors such as entanglements and vessel collisions (Kraus, 1990; Clapham et al., 1999; IWC, 2001a). Entanglements in fishing gear may represent a significant problem for the western population of North Pacific right whales, particularly given the present operation of Japanese salmon driftnet fisheries within the Russian EEZ inside the Okhotsk Sea.

The prognosis for the eastern North Pacific population is poor. This population is one of the most endangered populations of whales in the world and is also one of the most poorly studied (Clapham et al., 1999). A long-term monitoring programme is needed to better understand the conservation status of this population and to determine if it may be affected by any negative human interactions that require mitigation. This is especially needed because these whales are long lived, delay breeding, have a long reproductive cycle and have a small current population. Long-term monitoring will also allow better determination of the range of the summer feeding grounds in the eastern North Pacific. It is hoped that the increases reported in southern right whale populations, which were also heavily exploited during the $19^{\text {th }}$ century and then again by USSR whaling operations (during the late 1950s and 1960s) in the Southern Hemisphere (Tormosov et al., 1998), will be repeated by the right whale population in the eastern North Pacific.

\section{ACKNOWLEDGEMENTS}

We are greatly indebted to the late A.A. Berzin and to N.V. Doroshenko for their interest and efforts to ensure that the true soviet whaling catches of right whales in the North Pacific be made public. Cherry Allison kindly supplied information from the International Whaling Commission's database. The manuscript benefited greatly from thorough reviews by Jim Carretta and Randall Reeves. We thank A.M. Burdin, K. Mori, S. Uchida, Ted Sweeney, Heehyoung Lee, Geoffrey Patton and Saang-Yoon Hyun for help with translation of Russian and Korean material. Finally, we are grateful to S.I. Kornev, V.S. Nikulin, Joan Goddard, David Rugh, Diane Gendron, Jim Carretta, Sally Mizroch and Richard Rowlett for supplying data on recent sightings, strandings or photographs, and to John Wong for information from China.

\section{REFERENCES}

Anonymous. 1976. Japan. Progress report on whale research, June 1974 to May 1975. Rep. int. Whal. Commn 26:416-24.

Anonymous. 1977. Japan. Progress report on whale research, June 1975 to May 1976. Rep. int. Whal. Commn 27:88-91.

Anonymous. 1979. Japan. Progress report on cetacean research, June 1977-May 1978. Rep. int. Whal. Commn 29:117-20.
Anonymous. 1980. Japan. Progress report on cetacean research, June 1978 to May 1979. Rep. int. Whal. Commn 30:155-60.

Anonymous. 1981. Japan. Progress report on cetacean research, June 1979-May 1980. Rep. int. Whal. Commn 31:195-200.

Anonymous. 1983. Japan. Progress report on cetacean research, June 1981 to May 1982. Rep. int. Whal. Commn 33:213-20.

Anonymous. 1985. Japan. Progress report on cetacean research, June 1983 to April 1984. Rep. int. Whal. Commn 35:168-71.

Anonymous. 1987. Japan. Progress report on cetacean research, June 1985 to April 1986. Rep. int. Whal. Commn 37:172-5.

Anonymous. 1991. Japan. Progress report on cetacean research, May 1989 to May 1990. Rep. int. Whal. Commn 41:239-43.

Anonymous. 1992. Japan. Progress report on cetacean research, June 1990 to March 1991. Rep. int. Whal. Commn 42:352-6.

Berzin, A.A. 1982. Whale research in the Pacific Ocean. In: Investigations, Conservation and Rational Exploitation of Marine Mammals. Papers presented to the 8th All-Union Conference, Astrakhan. pp.24-26. [In Russian] [Abstract].

Berzin, A.A. and Doroshenko, N.V. 1981. Right whales of the Okhotsk Sea. Rep. int. Whal. Commn 31:451-5.

Berzin, A.A. and Doroshenko, N.V. 1982. Distribution and abundance of right whales in the North Pacific. Rep. int. Whal. Commn 32:381-3.

Berzin, A.A. and Rovnin, A.A. 1966. Distribution and migration of whales in the northeastern Pacific, the Bering and Chukotsk Seas. Izv. TINRO 58:179-209. [In Russian].

Berzin, A.A. and Vladimirov, V.L. 1989. Recent distribution and abundance of cetaceans in the Sea of Okhotsk. Sov. J. Mar. Biol. 15(2):84-90.

Berzin, A.A. and Yablokov, A., V. 1978. Number and population structure of the main exploited species of cetaceans in the World Ocean. Zool. Zh. 57(12):1771-85. [In Russian with English Summary].

Best, P.B. 1987. Estimates of the landed catch of right (and other whalebone) whales in the American fishery, 1805-1909. Fish. Bull. 85(3):403-18.

Blokhin, S.A. 1988. Results of the expedition of the 'Tungus' research-scouting vessel in the coastal waters of the Far Eastern seas in June-October 1986. pp. 23-35. In: N.S. Chernysheva (ed.) Scientific Research on Sea Mammals of the Northern Part of the Pacific Ocean in 1986-1987. VNIRO, Moscow. [In Russian].

Braham, H.W. and Rice, D.W. 1984. The right whale, Balaena glacialis. Mar. Fish. Rev. 46:38-44.

Brown, S.G. 1986. Twentieth-century records of right whales (Eubalaena glacialis) in the northeast Atlantic Ocean. Rep. int. Whal. Commn (special issue) 10:121-7.

Brueggeman, J.J., Grotefendt, R.A. and Erickson, A.W. 1984. Endangered whale abundance and distribution in the Navarin Basin of the Bering Sea during the ice-free period. pp. 201-36. In: B.R. Melteff and D.H. Rosenberg (eds.) Proceedings of the Workshop on Biological Interactions Among Marine Mammals and Commercial Fisheries in the Southeastern Bering Sea. University of Alaska, Alaska. Sea Grant Report 84-1. 300pp.

Brueggeman, J.J., Newby, T. and Grotefendt, R.A. 1986. Catch records of twenty North Pacific right whales from two Alaska whaling stations, 1917-1939. Arctic 39(1):43-6.

Carretta, J.V., Lynn, M.S. and Leduc, C.A. 1994. Right whale (Eubalaena glacialis) sighting off San-Clemente Island, California. Mar. Mammal Sci. 10(1):101-5.

Clapham, P.J., Young, S.B. and Brownell, R.L. 1999. Baleen whales: conservation issues and the status of the most endangered populations. Mammal Rev. 29:35-60.

Collet, R. 1909. A few notes on the whale Balaena glacialis and its capture in recent years in the North Atlantic by Norwegian whalers. Proc. Zool. Soc. Lond. 91-98:+27 plates.

DeBus, B. (ed.). 1975. Whalewatcher. American Cetacean Society, San Pedro, CA.

Doroshenko, N.V. 2000. Soviet whaling for blue, gray, bowhead and right whales in the North Pacific Ocean, 1961-1979. pp. 96-103. In: A.V. Yablokov and V.A. Zemsky (eds.) Soviet Whaling Data (1949-1979). Center for Russian Environmental Policy, Moscow. 408pp.

Du Pasquier, T. 1986. Catch history of French right whaling mainly in the South Atlantic. Rep. int. Whal. Commn (special issue) 10:269-74.

Evans, K. 1998. Endangered right whale sighted in sanctuary. News from the Monterey Bay National Marine Sanctuary (Spring 1988).

Fiscus, C.H. and Niggol, K. 1965. Observations of cetaceans off California, Oregon, and Washington. US Fish Wildl. Serv. Spec. Sci. Rep. Fish. 498:1-27. 
Fujise, Y., Kishiro, T., Zenitani, R., Matsuoka, K., Kawasaki, M. and Shimamoto, K. 1995. Cruise report of the Japanese whale research program under a Special Permit for North Pacific minke whales in 1994. Paper SC/47/NP3 presented to the IWC Scientific Committee, May 1995 (unpublished). 29pp. [Paper available from the Office of this Journal].

Fujise, Y., Iwasaki, T., Zenitani, R., Araki, J., Matsuoka, K., Tamura, T., Aono, S., Yoshida, T., Hidaka, H., Nibe, T. and Tohyama, D. 1996. Cruise report of the Japanese whale research program under a Special Permit for North Pacific minke whales in 1995 with the result of a preliminary analysis of data collected. Paper SC/48/NP3 presented to IWC Scientific Committee, June 1996 (unpublished). 39pp. [Paper available from the Office of this Journal].

Gaskin, D.E. 1987. Updated status of the right whale, Eubalaena glacialis, in Canada. Can. Field-Nat. 101(2):295-309.

Gendron, D., Lanham, S. and Carwardine, M. 1999. North Pacific right whale (Eubalaena glacialis) sighting south of Baja California. Aquat. Mamm. 25(1):31-4.

Gilmore, R.M. 1956. Rare right whale visits California. Pac. Discovery 9(4):20-5.

Gilmore, R.M. 1978. Right whale. pp. 62-9. In: D. Haley (ed.) Marine Mammals of the Eastern North Pacific and Arctic Waters. 1st. Edn. Pacific Search Press, Seattle. 256pp.

Goddard, P.D. and Rugh, D.J. 1998. A group of right whales seen in the Bering Sea in July 1996. Mar. Mammal Sci. 14(2):344-9.

Herman, L.M., Baker, C.S., Forestell, P.H. and Antinoja. 1980. Right whale Balaena glacialis sightings near Hawaii: a clue to the wintering grounds? Mar. Ecol. Prog. Ser. 2:271-5.

International Whaling Commission. 2001a. Report of the Workshop on Status and Trends of Western North Atlantic Right Whales. $J$. Cetacean Res. Manage. (special issue) 2:61-87.

International Whaling Commission. 2001b. Report of the Workshop on the Comprehensive Assessment of Right Whales: A worldwide comparison. J. Cetacean Res. Manage. (special issue) 2:1-60.

Ishikawa, H. (ed.). 1994. Stranding Records of Cetaceans from the Coasts of Japan. Geiken Soshio (No. 6), The Institute of Cetacean Research, Tokyo. 94pp. [In Japanese].

Ishikawa, H., Yuzu, S., Shimamoto, K., Bando, T., Ohshima, K., Kasai, H., Kinoshita, T., Mazushima, Y., Iwakami, H., Nibe, T., Hosoyama, T., Kuramochi, T., Numano, K. and Miyamoto, M. 1997. Cruise report of the 1997 Japanese Whale Research Program under a Special Permit for western North Pacific (JARPN) in 1997. Paper SC/49/NP9 presented to the IWC Scientific Committee, September 1997, Bournemouth (unpublished). 28pp. [Paper available from the Office of this Journal].

Ivashin, M.V. 1987. Some data on cetaceans casting ashore the Commander Islands. Paper SC/39/O 28 presented to the IWC Scientific Committee, June 1987 (unpublished). 14pp. [Paper available from the Office of this Journal].

Ivashin, M.V. and Rovnin, A.A. 1967. Some results of the Soviet whale marking in the waters of the North Pacific. Norsk Hvalfangsttid. 56(6): 123-35.

Johnson, T. 1982. A survivor at sea. Oceans 15(5):52.

Kasamatsu, F. and Miyashita, T. 1991. Guide Book for Whales and Dolphins in the North Pacific. University of Tokyo Press, Tokyo. 148pp. [In Japanese].

Kellogg, R. 1931. Whaling statistics from the Pacific coast of North America. J. Mammal. 12:73-7.

Klumov, S.K. 1962. Gladkie (Yaponskie) kity Tikhogo Okeana [The right whales in the Pacific Ocean]. Tr. Inst. Okeanol. Akad. Nauk SSSR 58:202-97. [In Russian].

Kornev, S.I. 1994. A note on the death of a right whale (Eubalaena glacialis) off Cape Lopatka (Kamchatka). Rep. int. Whal. Commn (special issue) 15:443-4.

Kraus, S.D. 1990. Rates and potential causes of mortality in North Atlantic right whales (Eubalaena glacialis). Mar. Mammal Sci. 6(4):278-91.

Kraus, S.D., Prescott, J.H., Knowlton, A.R. and Stone, G.S. 1986. Migration and calving of right whales (Eubalaena glacialis) in the western North Atlantic. Rep. int. Whal. Commn (special issue) 10:139-44.

Kuzmin, A.A. and Berzin, A.A. 1975. Distribution and current abundance of gray and right whales in the Far East Seas. pp. 121-61. Biological Resources of the Far East Seas. TINRO, Vladivostok.

LeDuc, R., Perryman, L., MacDonald, M., Hyde, J. and Brownell, J., RL. 2000. Progress report on the eastern North Pacific right whale research during July 1999 . Paper SC/52/OS13 presented to the IWC Scientific Committee, June 2000, in Adelaide, Australia. [Paper available from the Office of this Journal].

Matsuura, Y. and Maeda, K. 1942. Biological investigations of whales from the northern Pacific. Hogei-shiryo 9:44-5. [In Japanese].
Maury, M.F. 1852 et seq. Whale Chart of the World. Series F (Wind and Current Charts) Sheet 1 (Washington, 1852), sheets 2-4 (no date).

Maury, M.F. 1853. A chart showing the favourite resort of the sperm and right whale by M.F. Maury, LLD Lieut. US Navy. Constructed from Maury's Whale Chart of the World, by Robert H. Wyman, Lieut. USN by Authority of Commo: Charles Morris USN Chief of Bureau of Ordnance and Hydrography. Washington, 1 sheet.

Mitchell, E.D. 1979. Comments on magnitude of early catch of east Pacific gray whale (Eschrichtius robustus). Rep. int. Whal. Commn 29:307-14.

Mitchell, E.D. and Reeves, R.R. 2001. Aboriginal whaling for gray whales of the eastern North Pacific stock. J. Cetacean Res. Manage. (special issue)

Miyashita, T. and Kato, H. 1998. Recent data on the status of right whales in the NW Pacific Ocean. Paper SC/M98/RW11 presented to the IWC Special Meeting of the Scientific Committee towards a Comprehensive Assessment of Right Whales Worldwide, 16-25 March, Cape Town, South Africa. [Paper available from the Office of this Journal].

Miyashita, T., Okamura, H., Vladimirov, V.A. and Doroshenko, N.V. 2000. Cruise report of the Japan-Russia joint sighting survey in the Sea of Okhotsk in 1999. Paper SC/52/RMP4 presented to the IWC Scientific Committee, June 2000, in Adelaide, Australia. [Paper available from the Office of this Journal].

Miyazaki, N. and Nakayama, K. 1989. Records of cetaceans in the waters of the Amami Islands. Mem. Natl Sci. Mus. (Tokyo) 22:235-49.

Moore, S.E., Waite, J.M., Mazzuca, L.L. and Hobbs, R.C. 2001. Mysticete whale abundance and observations of prey associations on the central Bering Sea shelf. J. Cetacean Res. Manage. 2(3).

Mori, K., Sato, F. and Mochizuki, A. 1998. Recent observation records of the northern right whale in the waters of Ogasaw Ara (Bonin Islands), Japan. The World Marine Mammal Science Conference, Monaco, January 1998, Abstract Volume. pp. 93-94. [Available from Society for Marine Mammalogy, Lawrence, Kansas, USA]

Morris, B.F., Alton, M.S. and Braham, H.W. 1983. Living marine resources of the Gulf of Alaska. A resource assessment for the Gulf of Alaska/Cook Inlet proposed oil and gas lease sale 88. US Dept. Commerce, NOAA Tech. Memo NMFS F/AKR-5. 232pp.

Nasu, K. 1960. Oceanographic investigation in the Chukchi Sea during the summer of 1958. Sci. Rep. Whales Res. Inst., Tokyo 15:143-58. Ohsumi, S. and Wada, S. 1974. Report of the Scientific Committee, Annex N. Status of whale stocks in the North Pacific, 1972. Rep. int. Whal. Commn 24:114-26.

Omura, H. 1957. Report on two right whales caught off Japan for scientific purposes under Article VIII of the International Convention for the Regulation of Whaling. Norsk Hvalfangsttid. 46(7):374-90. [In Norwegian and English].

Omura, H. 1958. North Pacific right whale. Sci. Rep. Whales Res. Inst., Tokyo 13:1-52.

Omura, H. 1968. Right whale survey in the Okhotsk Sea. Geiken Tsushin 205:199-220. [In Japanese].

Omura, H. 1986. History of right whale catches in the waters around Japan. Rep. int. Whal. Commn (special issue) 10:35-41.

Omura, H. and Ohsumi, S. 1964. A review of Japanese whale marking in the North Pacific to the end of 1962, with some information on marking in the Antarctic. Norsk Hvalfangsttid. 54(4):90-112.

Omura, H., Ohsumi, S., Nemoto, R., Nasu, K. and Kasuya, T. 1969. Black right whales in the North Pacific. Sci. Rep. Whales Res. Inst., Tokyo 21:1-78.

Park, G. 1987. History of the Whaling of the Korean Peninsula. Deha Press, Pusan. 562pp. [In Korean].

Payne, R. 1986. Long term behavioral studies of the southern right whale (Eubalaena australis). Rep. int. Whal. Commn (special issue) 10:161-7.

Perryman, W.L., LeDuc, R. and Brownell, R.L., Jr. 1999. Progress report on eastern North Pacific right whale research during July 1998. Paper SC/51/CAWS36 presented to the IWC Scientific Committee, May 1999, Grenada, WI (unpublished). 10pp. [Paper available from the Office of this Journal].

Pike, G.C. and MacAskie, I.B. 1969. Marine mammals of British Columbia. Bull. Fish. Res. Board Can. 171:1-54.

Rae-Dupree, J. and Krikorian, G. 1990. Right rare sight. Los Angel. Times 18 May 1990, pp. B-1, B-8.

Reeves, R.R. and Leatherwood, S. 1985. Sightings of right whales (Eubalaena glacialis) in the eastern North Pacific. Paper SC/37/PS3 presented to the IWC Scientific Committee, June 1985 (unpublished). 7pp. [Paper available from the Office of this Journal].

Reeves, R.R., Leatherwood, S., Karl, S.A. and Yohe, E.R. 1985. Whaling results at Akutan (1912-39) and Port Hobron (1926-37), Alaska. Rep. int. Whal. Commn 35:441-57. 
Rice, D.W. and Fiscus, C.H. 1968. Right whales in the south-eastern North Pacific. Norsk Hvalfangsttid. 57(5):105-7.

Rowlett, R.A., Green, G.A., Bowlby, C.E. and Smultea, M.A. 1994. The first photographic documentation of a northern right whale off Washington State. Northwest. Nat. 75:102-4.

Rowntree, V., Darling, J., Silber, G. and Ferrari, M. 1980. Rare sighting of a right whale (Eubalaena glacialis) in Hawaii. Can. J. Zool. 58:309-12.

Salden, D.R. and Mickelsen, J. 1999. Rare sighting of a North Pacific right whale (Eubalaena glacialis) in Hawaii. Pac. Sci. 53:341-5.

Scammon, C.M. (ed.). 1874. The Marine Mammals of the Northwestern Coast of North America, Described and Illustrated: Together with an Account of the American Whale-Fishery. John H. Carmany and Co., San Francisco. X+319+vpp. [Reprinted in 1968 by Dover Publications, Inc., New York].

Scarff, J.E. 1986a. Historic and present distribution of the right whale (Eubalaena glacialis) in the eastern North Pacific south of $50^{\circ} \mathrm{N}$ and east of $180^{\circ}$ W. Rep. int. Whal. Commn (special issue) 10:43-63.

Scarff, J.E. 1986b. Occurrence of the barnacles Coronola diameda, C. reginae and Cetopirus complanatus (Cirripedia) on right whales. Sci. Rep. Whales Res. Inst., Tokyo 37:129-53.

Scarff, J.E. 1991. Historic distribution and abundance of the right whale (Eubalaena glacialis) in the North Pacific, Bering Sea, Sea of Okhotsk and Sea of Japan from the Maury Whale Charts. Rep. int. Whal. Commn 41:467-89.

Scarff, J.E. 2001. Preliminary estimates of whaling-induced mortality in the 19th century North Pacific right whale (Eubalaena japonicus) fishery, adjusting for struck-but-lost whales and non-American whaling. J. Cetacean Res. Manage. (special issue) 2:261-268.

Shino, T. 1932. Result of winter whaling off Akkeshi. Shisuikaishi 27(9):881-962. [In Japanese].

Sleptsov, M.M. 1952. Kittobrazye dal'ne ostochnykh morei [Whales of the Far East]. Izv. TINRO 38. [In Russian].

Sleptsov, M.M. 1955. Biology of whales and the whaling fishery in Far Eastern seas. 'Pishch. Prom.', Moscow. [Translation with comments and conclusions only by Fisheries Research Board Canada, Translation Service 118, 6pp]. [In Russian].

Slijper, E.J., van Utrecht, W.L. and Naaktgeboren, C. 1964. Remarks on the distribution and migration of whales, based on observations from Netherlands ships. Bijdr. Dierkd. 34:3-93.

Terry, W.M., 1950. Japanese whaling industry prior to 1946 . General Headquarters Supreme Commander for the Allied Powers, Natural Resources Section, Report No. 126. 47pp.

Tomilin, A.G. 1957. Zveri SSSR i Prilezhasfchikh Stran. Zveri Vostochnoi Evropy i Severnoi Azii. Izdatel'stvo Akademi Nauk SSSR, Moscow. 756pp. [Translated in 1967 as Mammals of the USSR and Adjacent Countries. Mammals of Eastern Europe and Adjacent Countries. Vol. IX. Cetacea by the Israel Program for Scientific Translations, Jerusalem, 717pp.][In Russian].

Tonnessen, J.N. and Johnsen, A.O. 1982. The History of Modern Whaling. C. Hurst \& Co., London. i-XX+798pp.

Tormosov, D.D., Mikhalev, Y.A., Best, P.B., Zemsky, V.A., Sekiguchi, K. and Brownell Jr, R.L. 1998. Soviet catches of southern right whales, Eubalaena australis, 1951-1971; biological data and conservation implications. Biol. Conserv. 86(2):185-97.

Townsend, C.H. 1935. The distribution of certain whales as shown by logbook records of American whaleships. Zoologica (NY) 19(1-2):1-50+6 maps.
Tynan, C.T. 1998. Critical habitat and abundance estimation or right whales in the southeast Bering Sea. Paper SC/50/CAWS18 presented to the IWC Scientific Committee, April 1998 (unpublished). [Paper available from the Office of this Journal].

Tynan, C.T. 2001. Ecological refuge for endangered North Pacific right whales on the southeastern Bering Sea shelf. Nature, Lond.

Vladimirov, V.L. 1994. Recent distribution and abundance of whales in Russian Far-Eastern seas. Russian J. Mar. Biol. 20:1-9.

Vladimirov, V.L. 2000. Present distribution, population numbers and structure in whales of the Far East seas. pp. 104-22. In: A.V. Yablokov and V.A. Zemsky (eds.) Soviet Whaling Data (1949-1979). Center for Russian Environmental Policy.

Wada, S. 1975. Report of the Scientific Committee, Annex L. Indices of abundance of large-sized whales in the North Pacific in 1973 whaling season. Rep. int. Whal. Commn 25:129-65.

Wada, S. 1977. Indices of abundance of large-sized whales in the North Pacific in the 1975 whaling season. Rep. int. Whal. Commn 27:189-94.

Wada, S. 1978. Indices of abundance of large-sized whales in the North Pacific in the 1976 whaling season. Rep. int. Whal. Commn 28:319-24.

Wada, S. 1979. Indices of abundance of large sized whales in the North Pacific in the 1977 whaling season. Rep. int. Whal. Commn 29:253-64.

Wada, S. 1981. Japanese whaling and whale sighting in the North Pacific 1979 season. Rep. int. Whal. Commn 31:783-92.

Wang, P. 1978. Studies on the baleen whales in the Yellow Sea. Acta Zool. Sin. 24(3):269-77.

Wang, P. 1984. Distribution of cetaceans in Chinese waters. Chin. J. Zool. 6:52-6. [Translated in 1985 by C.H. Perrin and edited by W.F. Perrin, NMFS, SWFC Admin. Rep. LJ-85-24, 11pp.] [In Chinese].

Wang, P. 1988. Cetacea. p. 342. In: Z. Xiao (ed.) Fauna Liaoningica. Liaoning Science and Technology Press, Shenyang, China. pp119162.

Webb, R.L. 1988. On the Northwest, Commercial Whaling in the Pacific Northwest 1790-1967. University British Columbia Press, Vancouver. xxi+425pp.

Woodhouse, C.D. and Strickley, J. 1982. Sighting of northern right whale (Eubalaena glacialis) in the Santa Barbara channel. J. Mammal. 63(4):701-2.

Yablokov, A.V. 1994. Validity of whaling data. Nature, Lond. 367(6459):108.

Yamamoto, Y. and Hiruta, H. 1978. Stranding of a black right whale at Kumomi, southwestern coast of Izu Peninsula. Sci. Rep. Whales Res. Inst., Tokyo 30:249-51.

Zemsky, V.A., Berzin, A.A., Mikhalev, Y.A. and Tormosov, D.D. 1995. Report of the sub-committee on Southern Hemisphere baleen whales. Appendix 3. Soviet Antarctic pelagic whaling after WWII: Review of actual catch data. Rep. int. Whal. Commn 45:131-5.

Zenkovich, B.A. 1934. Whaling in the Kamchatka and Bering Seas during the 1933 season. Rybn. Khoz. 1-2:113-8. [In Russian].

Zenkovich, B.A. 1955. Chapter 1 . A brief history of whaling in prerevolutionary Russia and an account of present-day whaling in the USSR. pp. 5-29. In: S.E. Kleinenberg and T.I. Makarova (eds.) The Whaling Industry of the Soviet Union. Research Institute of Marine Fisheries and Oceanography (VNIRO), Moscow. [English Translation by Israeli Program for Scientific Translation, 1968]. [In Russian]. 